\title{
Detectors for low energy electron cooling in RHIC
}

\author{
F.S. Carlier
}

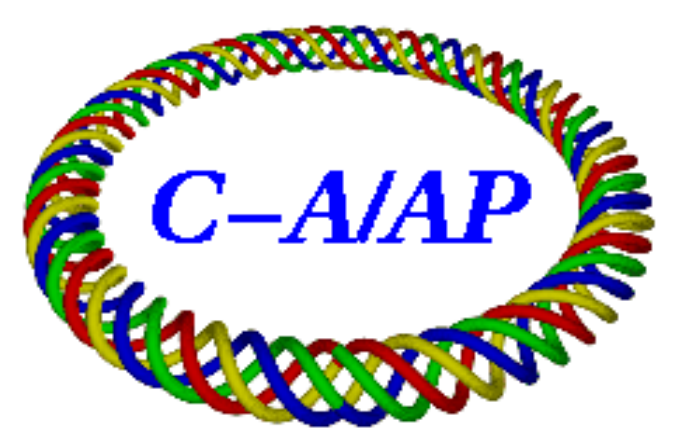

\section{Collider-Accelerator Department Brookhaven National Laboratory \\ Upton, NY 11973}

\author{
U.S. Department of Energy \\ Office of Science, Office of Nuclear Physics
}

Notice: This document has been authorized by employees of Brookhaven Science Associates, LLC under Contract No. DE-SC0012704 with the U.S. Department of Energy. The United States Government retains a nonexclusive, paid-up, irrevocable, world-wide license to publish or reproduce the published form of this document, or allow others to do so, for United States Government purposes. 


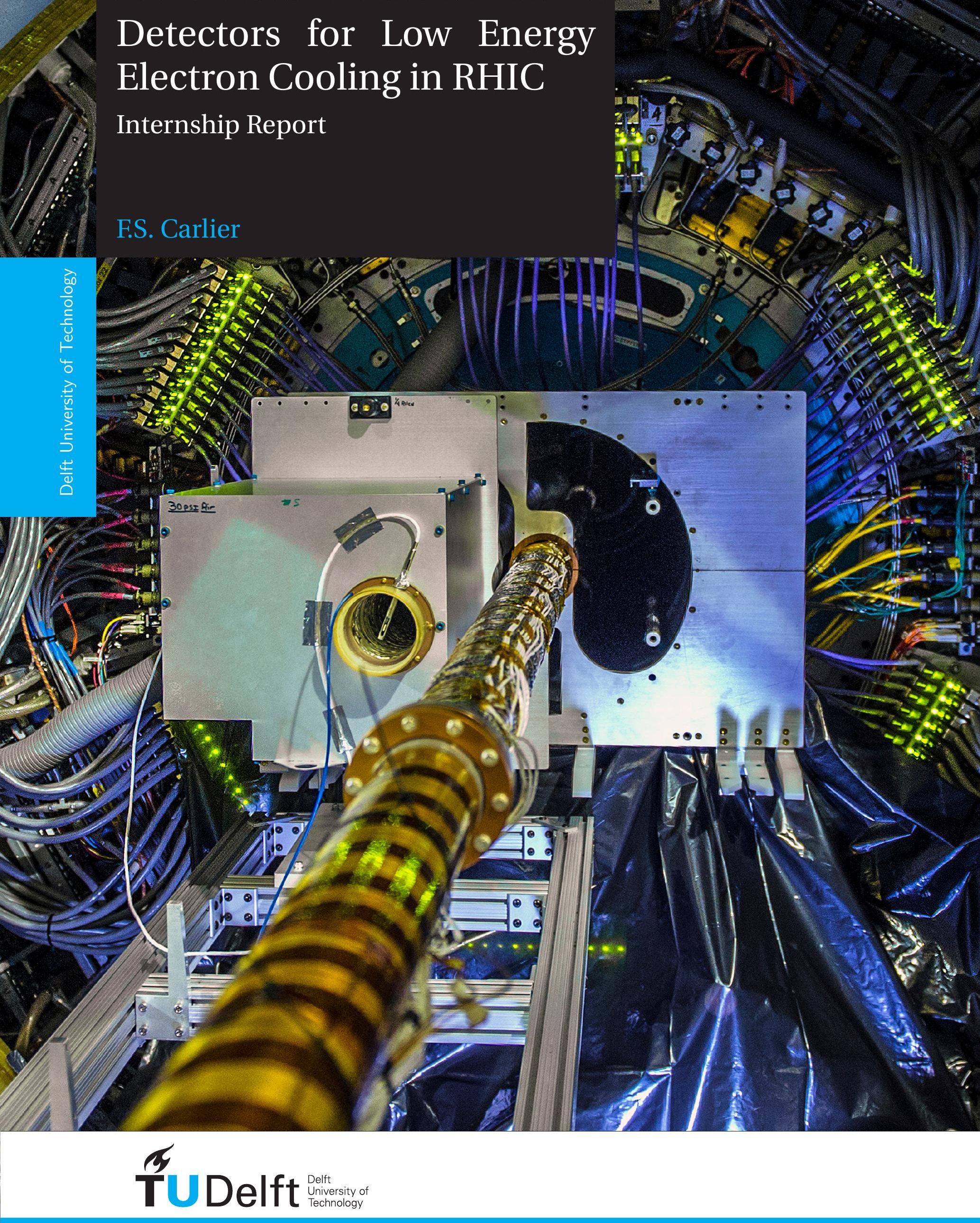




\section{Contents}

1 Introduction 2

2 Radiative Recombination in the Electron Cooler 3

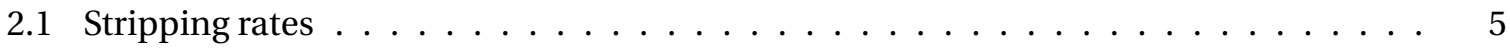

3 Radiative Recombination Monitors $\quad 8$

3.1 Schottky Spectra . . . . . . . . . . . . . . . . . . . . . . . . 8

3.2 Excitation of $\mathrm{Au}^{78+}$ using Induced Tune Shift $\ldots \ldots \ldots \ldots \ldots$

3.2.1 Beam Transfer Function . . . . . . . . . . . . . . . . . . . . . . . . 9

3.2 .2 Excitation using AC Dipole. . . . . . . . . . . . . . . . . . 10

3.3 Lattice with large dispersion \& small $\beta$-function. . . . . . . . . . . . . . . 11

3.3 .1 Lattice for BLUE Beam . . . . . . . . . . . . . . . . . . . . . . . . . . . . . 12

3.3.2 Lattice for YELLOW Beam . . . . . . . . . . . . . . . . . . . . . . . . 14

3.3.3 Detectors for Recombined Ions in the Arcs . . . . . . . . . . . . . . . . . . 14

4 Alternatives to Recombination Monitors 20

4.1 Direct detection of Electron Cooling with Schottky System . . . . . . . . . . . . . . . 20

4.2 Recombination of Deuteron. . . . . . . . . . . . . . . . . . . . . 20

5 Conclusions 23

A Appendix A $\quad 25$

B Appendix B $\quad 27$

Bibliography $\quad 31$ 
The studies presented in this chapter were collaborative efforts. Many people contributed to the results presented. Notable acknowledgements should go to Wolfram Fischer, Mike Blaskiewicz, Alexei Fedotov, Peter Thieberger, Guillaume Robert-Demolaize, Michiko Minty, Christoph Montag and Angelika Drees. 


\section{Introduction}

Low energy operation of RHIC is of particular interest to study the location of a possible critical point in the QCD phase diagram [1-4]. The performance of RHIC at energies equal to or lower than $10 \mathrm{GV} /$ nucleon is limited by nonlinearities, Intra-Beam Scattering (IBS) processes and space-charge effects. To successfully address the luminosity and ion store lifetime limitations imposed by IBS the method of electron cooling has been envisaged. During electron cooling processes electrons are injected along with the ion beam at the nominal ion bunch velocities. The velocity spread of the ion beam is reduced in all planes through Coulomb interactions between the cold electron beam and the ion beam. The electron cooling system proposed for RHIC will be the first of its kind to use bunched beams for the delivery of the electron bunches, and will therefore be accompanied by the necessary challenges. The designed electron cooler will be located in IP2. The electron bunches will be accelerated by a linac before being injected along side the ion beams. Thirty consecutive electron bunches will be injected to overlap with a single ion bunch. They will first cool the yellow beam before being extracted turned by 180-degrees and reinjected into the blue beam for cooling. As such, both the yellow and blue beams will be cooled by the same ion bunches. This will pose considerable challenges to ensure proper electron beam quality to cool the second ion beam. Furthermore, no ondulator will be used in the electron cooler so radiative recombination between the ions and the electrons will occur.

Alignment of the ion and electron beams as well as the matching of the velocities between both bunches is crucial to deliver the desired cooling efficiencies. The electron cooling force greatly decreases for increasing relative energy deviation between both beams. However, direct measurements of the absolute energy of the electron beam will be challenging and are predicted to be accurate up to $10^{-3}$. Energy matching between the ion and electron bunches to achieve cooling is therefore not guaranteed. As such, various tuning and monitoring methods will need to be developped to ensure the proper cooling conditions. Among those, monitoring of recombination rates in the electron cooler is believed to be the most viable secondary cooling detector. Recombination rates of ions with electrons are less sensitive to energy mismatch than cooling efficiencies, and can therefore function as a easier to detect signal to tune the energies in the regime of large relative energy deviation in which electron cooling is weak, $\frac{d E}{E}>0.1 \%$. Monitoring of recombination rates in the electron cooler will provide a good understanding of the beam overlap and energy mismatch of the ion and electron beams.

This study was performed as part of the Collider Accelerator Department (C-AD) at the Brookhaven National Laboratory (BNL) on behalf of the Low Energy RHIC Electron Cooling (LEReC) project. The report addresses the production rates of recombined ions as well as the various methods available to monitor recombination rates. Furthermore, it highlights the challenges left for the detection of electron cooling and proposes procedures for the commissioning of Low Energy RHIC operations. 


\section{Radiative Recombination in the Electron Cooler}

A small portion of the fully stripped golds ions will recombine with electrons in the electron cooler through radiative recombination processes as given in Eq. (2.1). This will result in a population of non-fully stripped hydrogen-like gold ions that propagate in the accelerator [5] [6] [7] [8]. Further recombination to $A u^{77+}$ or higher states is also possible, but occurs at such low probabilities that it has been neglected for the purpose of this study.

$$
\mathrm{Au}^{79+}+e^{-} \rightarrow \mathrm{Au}^{78+}+\gamma
$$

The recombination of gold ions with electrons is currently believed to be one of the most viable method to tune the energies of the ion and electron beams to approach cooling conditions when the velocities are mismatched. Recombination rates of fully stripped gold ions in electron bunches will therefore be crucial to successfully adjust the electron beam energies. Estimates of recombination rates are commonly calculated using the methods presented in [5]. Previous estimates for the LEReC used the asymptotic formulae in [5] to approximate recombination rates as a function of relative energy deviations [9] [10]. This report presents a more thorough approach to estimate the recombination rates and lifetimes as a function of the relative beam velocities of the ion and electron bunch.

The capture cross section in the ion rest frame for an electron is given to good approximation by [5].

$$
\sigma=A\left(\frac{h \nu_{0}}{E}\right)\left[\ln \sqrt{\frac{h v_{0}}{E}}+0.1402+0.525\left(\frac{E}{h \nu_{0}}\right)^{\frac{1}{3}}\right]
$$

where $E$ is the electron kinetic energy, $h$ is the Planck constant, $A=\frac{4}{3 \sqrt{3} \pi} \frac{h e^{2}}{\epsilon_{0} m_{e}^{2} c^{3}}=2.11 \cdot 10^{-22}$ and $h v_{0}=13.6 Z^{2} \mathrm{eV}$ is the ground state binding energy. The capture cross section may be expressed as a function of the transverse and longitudinal electron velocities by,

$$
\sigma=\frac{2 A h v_{0}}{m_{e}} \frac{1}{\left(v_{\|}^{2}+v_{\perp}^{2}\right)}\left[\frac{1}{2} \ln \frac{2 h v_{0}}{m_{e}}-\frac{1}{2} \ln \left(v_{\|}^{2}+v_{\perp}^{2}\right)+\gamma_{1}+\gamma_{2}\left(\frac{m_{e}}{2 h v_{0}}\right)^{\frac{1}{3}}\left(v_{\|}^{2}+v_{\perp}^{2}\right)^{\frac{1}{3}}\right]
$$

where $m_{e}$ is the electron mass, $v_{\perp}$ and $\nu_{\|}$are the particle velocities perpendicular and paralellel to the bunch velocity in the ion rest frame and $\gamma_{1}=0.1402$ and $\gamma_{2}=0.525$ are constants. The recombination rate coefficient $\alpha_{r}$ is obtained by integration over the velocity distributions and the cross section. 


\begin{tabular}{|c|c|}
\hline Parameter & value \\
\hline$\gamma$ & 4.1 \\
\hline$\frac{\delta p}{p}$ & $5 \cdot 10^{-4}$ \\
\hline$\theta_{\perp}$ & $150 \mu \mathrm{rad}$ \\
\hline$n_{e f f}$ & $3.22 \cdot 10^{12} \mathrm{~m}^{-3}$ \\
\hline$\eta$ & $18 / 3833=0.004696$ \\
\hline
\end{tabular}

Table 2.1: Beam parameters used for calculations of recombination rates.

$$
\alpha_{r}\left(v_{r e l}\right)=\int \sigma(v) v f_{e}\left(\mathbf{v}_{e}, v_{r e l}\right) f_{i}\left(\mathbf{v}_{i}, v_{r e l}\right) \mathrm{d}^{3} v
$$

where $f_{e}\left(\mathbf{v}_{e}, v_{r e l}\right)$ and $f_{i}\left(\mathbf{v}_{i}, v_{r e l}\right)$ are the Maxwellian velocity distributions of respectively the electron- and the ion bunch. Both distributions are dependent on the relative velocities between the ion and electron bunches $\left(v_{r e l}\right)$. The full derivations of the integrals are presented in Appendix A. The beam lifetime as a result of the recombination process in the cooling section is obtained from,

$$
\tau=\frac{\gamma^{2}}{n_{e f f} \alpha_{r} \eta}
$$

where $\eta=\frac{l_{e}}{C}$, the fraction of the accelerator length occupied by the electron cooler, and where effective electron density is given by,

$$
n_{e f f}=\frac{N_{e}}{(2 \pi)^{\frac{3}{2}} \sigma_{e, z}^{2} \sigma_{e, s} \gamma} \cdot \frac{30 \cdot \sigma_{e, s}}{\sigma_{i, s}}
$$

where $\sigma_{e, s}$ and $\sigma_{i, s}$ are the longitudinal RMS bunch lengths of the electron and ion bunches respectively, $\sigma_{e, z}$ is the transverse RMS size of the electron bunches, and where $\eta$ is the fraction of the accelerator length occupied by the electron cooler. The relative energy in laboratory frame is then determined using:

$$
\frac{\Delta E}{E}=\frac{\gamma_{\text {elec }}-\gamma_{\text {nominal }}}{\gamma_{\text {nominal }}-1}
$$

The results for the radiative recombination rate as function of relative energies between the ion and electron beams are presented in figure 2.1. The left plot shows the recombination rates at small relative energies where detection of the cooling with the Schottky system will be possible. Maximal recombination, with $\alpha_{r}=3.1 \cdot 10^{-14} \mathrm{~m}^{3} \mathrm{~s}^{-1}$, occurs for matching velocities between the two bunches. The rate quickly decreases for increasing relative energies. Results of recombination rates for larger relative energy deviations are shown in the right plot. The gradient clearly decreases for increasing energy deviation.

The ion lifetimes calculated using Eq. (2.5) are presented in figure 2.2. The left plot shows the lifetimes for relative energies $\frac{d E}{E}<0.1 \%$, while the right plot shows the lifetimes extended to larger relative energy deviations, $\frac{d E}{E}<1 \%$. The lifetime dependency to large relative energies is almost linear. As the population of $\mathrm{Au}^{78+}$ present in RHIC will be directly dependent on the lifetime, this will provide a proper measurable to tune the energies.

The obtained lifetimes are larger than estimates made using the asymptotic formulae in [5]. Though larger, these results are still sufficient to use recombination to monitor the cooling conditions in the electron cooler.

The dependence on the transverse relative displacement between the ion and the electron beams has been roughly estimated. It is assumed that both beams are straight and parallel. This is not the 

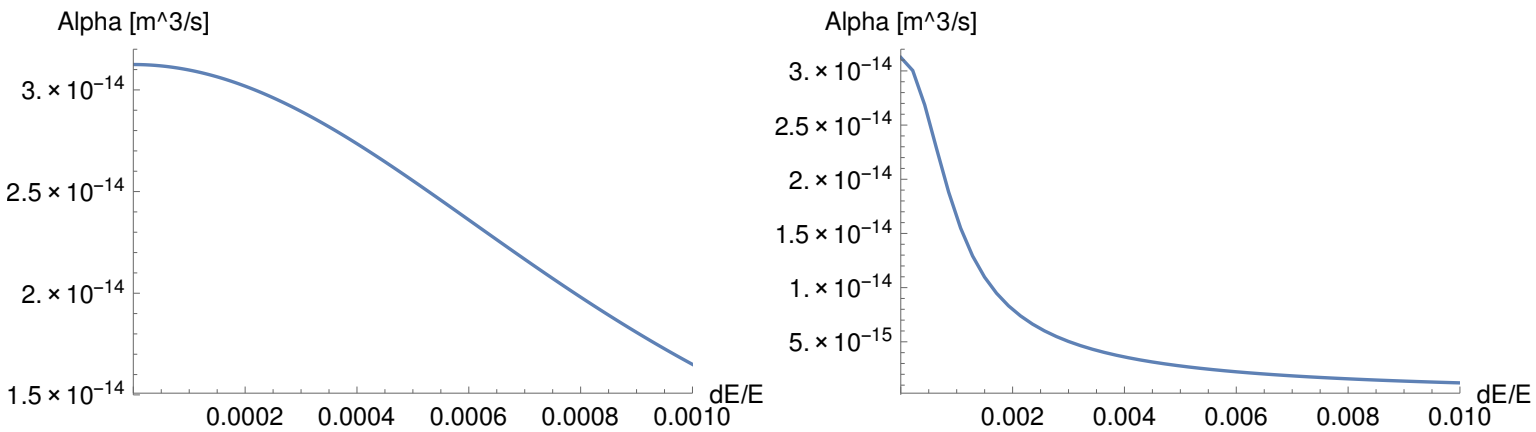

Figure 2.1: Radiative recombination rate as as a function of relative energy between the ion and electron bunches.
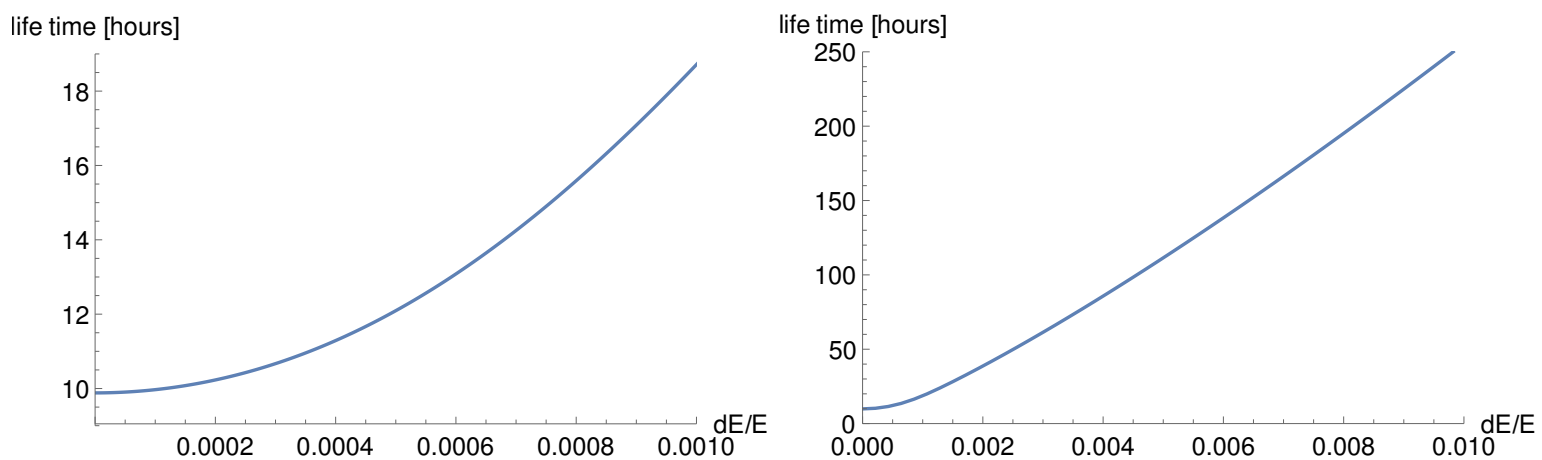

Figure 2.2: Lifetime of ions including the longitudinal overlap of the ion and electron beams.

case in general as the electron beam will not be exactly straight throughout the cooling section. However, relative angles between the beams have limited impact on the recombination. In this straight beams approximation the recombination rate will be linearly dependent on the overlapping area between the ion and electron beam. The area is obtained through integration of the area between two overlapping circles with radii $\sigma_{e, \perp}$ and $\sigma_{i, \perp}$. The recombination rate will be maximal for zero displacement $(d=0)$, and will decrease for finite displacements until the beams do not overlap. Figure 2.3 shows the decrease of recombination with relative distance.

\subsection{Stripping rates}

The total population of recombined ions inside the accelerator will depend on the production rate as well as the loss rate. Part of the ions will experience ionization due to scattering with the residual gas in the accelerator. Ionization of $\mathrm{H}$ - and He-like heavy projectile ions (high $\mathrm{Z}$ ) at relativistic ener-

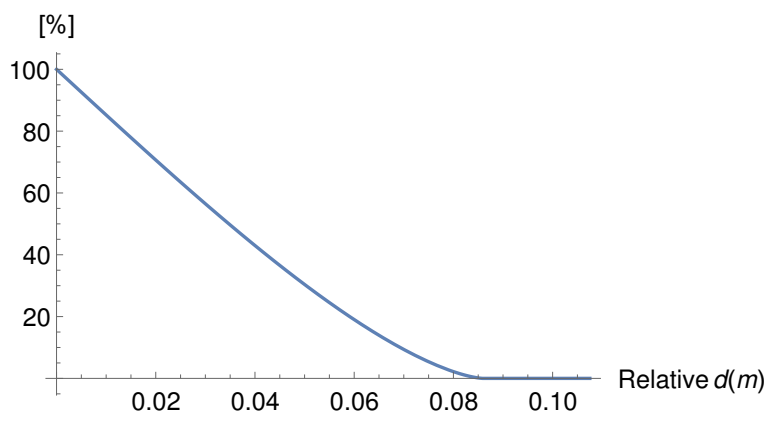

Figure 2.3: Percentage of maximum recombination rate as a function of transverse distance between the ion and electron beam. 


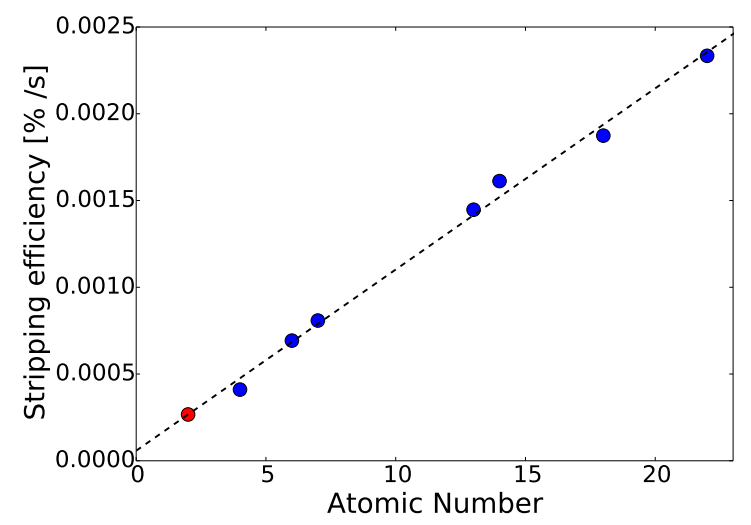

Figure 2.4: Stripping efficiencies per second for various elements. The efficiency for hydrogen is obtained by from a linear fit of the results and extrapolating to $Z=2$.

gies is best described by first order perturbation theory such as the plane wave Born approximation (PWBA) [11]. The obtained cross section for such an ionization process is given by,

$$
\sigma_{\text {ion }}=\sigma_{0} f\left(\frac{v}{v_{K}}\right)=4 \pi a_{0}^{2} \frac{Z_{T}^{2}}{Z_{P}^{2}} f\left(\frac{v}{v_{K}}\right)
$$

where $a_{0}$ is the Bohr radius, $Z_{T}$ and $Z_{P}$ denote the nuclear charge of the target and projectile respectively, and $\sigma_{i o n}$ is a function of the projectile velocity $v$ and has a maximum at $v=v_{K}$, the velocity of the active K-shell electron. The resulting population of ions due to ionization in residual gasses can be calculated in LISE++ [12]. LISE++ requires an effective target thickness to simulate these collisions. The effective target thickness is dependent on the pressure $(P)$, temperature $(T)$ and gas composition, and is determined using the ideal gas law. Furthermore, only the warm sections of the RHIC are used.

$$
\eta_{t h}=\frac{P}{k T} m_{H^{2}} l_{w a r m} n \cdot 10^{2} \mathrm{mg} / \mathrm{cm}^{2}
$$

where $k$ is the Boltzmann constant, $m_{H^{2}}$ is the hydrogen molecule mass, $l_{\text {warm }}$ is the total length of warms sections in RHIC, and $n$ is the number of turns. The following effective target thickness is obtained.

$$
\eta_{t h}=0.075 \cdot 10^{-3} \mathrm{mg} / \mathrm{cm}^{2}
$$

It should be noted that the widely used cross sections proposed by Franzke [13] is not valid for relativistic energies and should therefore not be used.

The Low-Energy RHIC will operate as low as $\gamma=4.1$, which corresponds to $E=3.8 \mathrm{GeV} /$ nucleon. Using these parameters the stripping efficiency is determined using LISE++/CHARGE. Simulations with LISE++ are limited to collisions with Be- or heavier targets. As such collisions with Hydrogen gas can not be directly simulated. Stripping efficiencies of a range of different targets have been determined, the results have been extrapolated to obtain an estimate of the stripping efficiency for hydrogen. The stripping efficiency for hydrogen is obtained by fitting the efficiencies of the available targets and is estimated at $2.67 \cdot 10^{-4} \%$

For completeness both the ion populations have been calculated as a function of running time through a balance of recombination and stripping rates. The results are presented in figure 2.5 for the time-span of one hour. The population of recombined ions grows linearly over time. After one hour $8 \%$ of the gold ions will have recombined with an electron. After 1 minute $8 \%$ of the ions will 


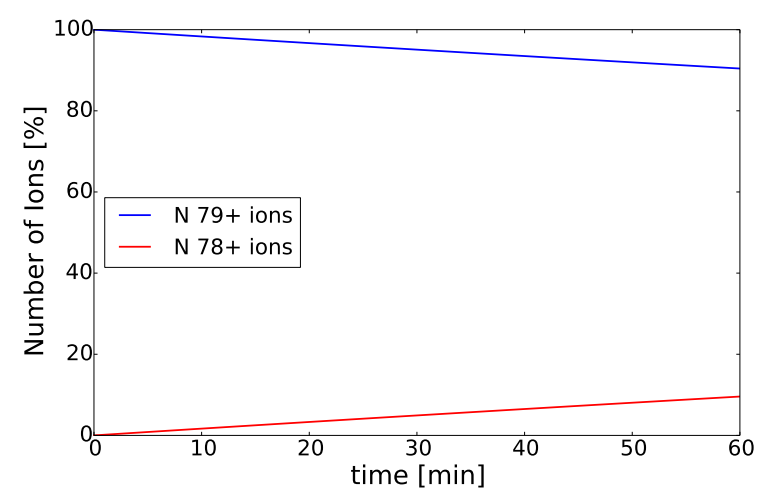

Figure 2.5: The evolution of the recombined ion and fully stripped ion populations over the time-span of one hour. The population of $\mathrm{Au}^{78+}$ ions after one hour is approximately $9 \%$ of the total initial gold ions. Note that this is for parameters in Tab. 2.1 and matched gammas.

have recombined with an electron. This is small but sufficient to detect with a monitor such as the BTF or large dispersion lattice, which will be discussed in section 3 


\section{Radiative Recombination Monitors}

Several methods have been considered to detect the recombined ions in RHIC, each of which uses a different property of these recombined ions. First, detection with the Schottky system using the difference in revolution frequency is considered. Later the tune shift between the two different populations is used when looking at using the BBQ or AC-dipoles. Lastly, the charge difference is used to separate the two ion populations in large dispersive regions.

\subsection{Schottky Spectra}

The Schottky system may potentially be used to detect the recombined ions due to their slightly different revolution frequency. Generally, the change in revolution frequency is dependent on the change in circumference and the change in momentum.

At this point it is important to note that the ions subject to recombination will have an unchanged velocity and therefore an unchanged momentum. In the process of capturing one electron, the ion velocity does not change. Its momentum will therefore only increase due to the additional electron mass, which is negligible at this level. The change in revolution frequency of $\mathrm{Au}^{78+}$ is therefore only dependent on the change in circumference due to the decreased charge. Furthermore, as the momentum is unchanged the recombined ions will not leave the bucket. Previous assumptions that recombined ions would leak into the abort gap and could be kicked resonantly are therefore dismissed.

The frequency shift is determined from the momentum compaction factor $\alpha_{c}=1 / \gamma_{T}^{2}$ and the charge difference of the $\mathrm{Au}^{78+}$ ions, $\frac{\Delta q^{\prime}}{q^{\prime}}=-1 / 78$ [14].

$$
\Delta C=\oint \frac{D(s)}{\rho} d s \frac{\Delta q}{q}
$$

and the momentum compaction factor is given by,

$$
\begin{aligned}
& \alpha_{c} \equiv \frac{1}{C} \frac{d \Delta C}{d(\Delta q / q)}=\frac{1}{C} \oint \frac{D(s)}{\rho} d s \\
& \frac{\Delta f}{f}=\frac{\Delta C}{C}=\alpha_{c} \frac{\Delta q}{q}=-2.4 \cdot 10^{-5}
\end{aligned}
$$

The acceptance of the bucket is given by frequency spread of the unrecombined ions:

$$
\sigma_{f}=-\left(\frac{1}{\gamma_{T}^{2}}-\frac{1}{\gamma^{2}}\right) \delta=2.9 \cdot 10^{-5}
$$




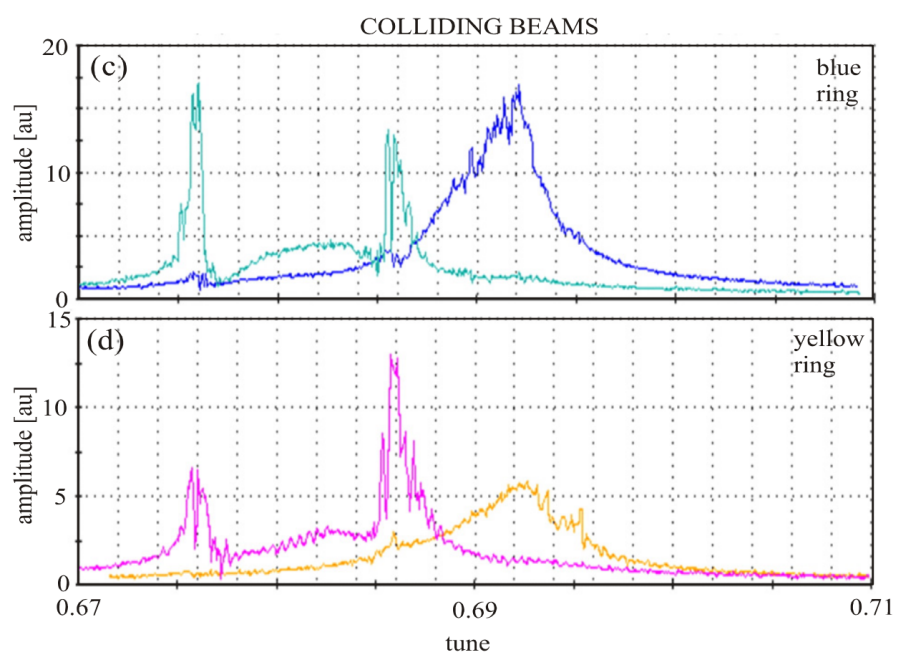

Figure 3.1: Results of BTF measurements presented in [15].

It may be concluded that the revolution frequency of the recombined gold ions will be within the frequency spread and will therefore not be detectable.

\subsection{Excitation of $\mathrm{Au}^{78+}$ using Induced Tune Shift}

Due to the change of charge of the recombined gold ions, these ions will experience a tune shift. Depending on the chromaticity of the machine this tune shift can become quite significant. RHIC commonly runs with a first order chromaticity of $Q=2$. Recombined gold ions will have a relative charge difference of $\Delta q / q=1 / 79$.

$$
\begin{aligned}
\Delta Q_{z} & =\frac{1}{4 \pi} \oint \beta_{z} \Delta K_{z} d s \\
& =\frac{1}{4 \pi} \oint \beta_{z} K_{z} d s \frac{\Delta q}{q} \\
& =Q^{\prime} \frac{\Delta q}{q}
\end{aligned}
$$

The resulting tune shift is $\Delta Q=-0.025$ for a nominal chromaticity of 2 . The ions travel through the accelerator with a momentum spread of $\Delta p / p=5 \cdot 10^{-4}$, which yields a tune spread of $10^{-3}$. The tune spread is much smaller than the induced tune shift arising from the charge difference. As such, the recombined ions may be excited independently of the main ion population. It should be noted that this will also depend on the tune spread induced by the space-charge at low energies.

\subsubsection{Beam Transfer Function}

The induced tune shift of the recombined ions opens the door for measuring the tune distribution using the beam transfer function. The beam transfer function offers a very precise measurement of the tune distribution in the beam, and could therefore discern the two different ion populations. It has been used previously to succesfully detect the $\pi$-modes arising during collision in RHIC [15]. The response of the BTF system is linearly dependent on the current and thus on the number of excited particles. Furthermore, it will also depend linearly on the oscillation amplitude [16].

$$
\langle x\rangle(t)=\frac{A}{2 \omega_{x}}\left[\cos (\Omega t) \text { P.V. } \int d \omega \frac{\rho(\omega)}{\omega-\Omega}+\pi \rho(\Omega) \sin (\Omega t)\right]
$$



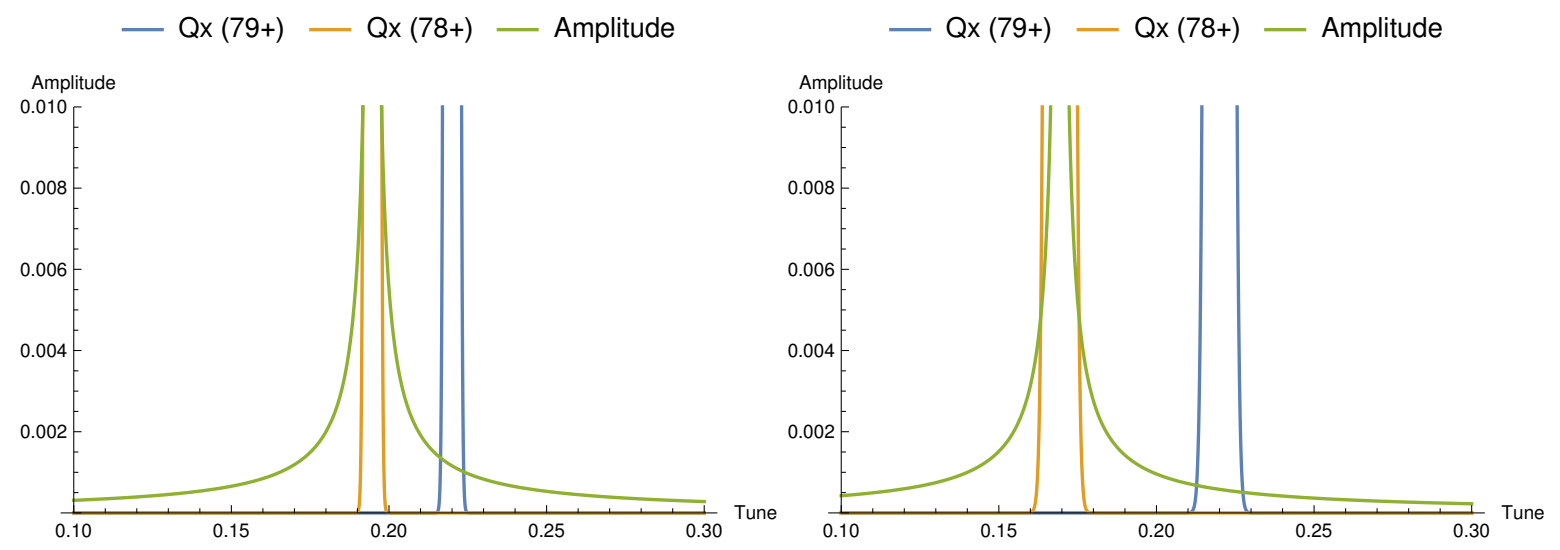

Figure 3.2: The induced oscillation amplitudes induced by the AC dipole driven at the Au78 tune are shown in green. The tune density distributions for the ion bunch (blue) and the recombined ions (yellow) are also given. The left plot shows the results for $Q^{\prime}=2$, while the right plot show the the same analysis for $Q^{\prime}=4$.

A quick estimate can be made to determine the needed oscillation amplitude for the $\mathrm{Au}^{78+}$ ions based on results of the proton runs. The signal amplitudes are proportional to the oscillation amplitude $(x)$ and current $(I)$. An estimate can be made by equating both signals from protons and ions as in Eq. (3.8). Recent measurements with $10^{11}$ protons and oscillation amplitudes of $100 \mathrm{~nm}$, yielded precise measurements of tune distributions. The amount of recombined ions after 1 minute is approximately $0.17 \%$.

$$
\begin{aligned}
(x I)_{\text {protons }} & =(x I)_{\text {ions }} \\
x_{\text {ions }} & =150 \mu \mathrm{m}
\end{aligned}
$$

Such amplitudes are reachable with the current setup of the BTF system. As the tune shift between the recombined ions and the main bunch is much larger than the tune spread of the beam, measurements of using the BTF should not be perturbed with excited Au79 ions.

\subsubsection{Excitation using AC Dipole}

o The oscillation amplitude of excited beams using an AC dipole is given by [17],

$$
z(s)=\frac{B_{m} L \sqrt{\beta(s) \beta_{0}}}{4 \pi B \rho} \frac{1}{\delta}
$$

with $\delta=\left|v_{m}-v_{z}\right|$. By exciting on the resonance $\delta=0$ it is possible to generate large oscillation amplitudes for the recombined ions while only producing small excitations in the main ion bunch. The recombined ions can then be lost at a collimator where the losses can be monitored. The induced oscillation amplitudes are shown in figure 3.2 in green, while the distribution of the recombined ions and of the fully stripped ions are shown in yellow and blue respectively. The left plot shows the results assuming a chromaticity of 2 , while in the right plot the chromaticity is doubled to 4 .

Taking the threshold to be $4 \cdot \sigma_{\text {beam }}$, and integrating the distributions over the limits that enclose the amplitudes $x>4 \sigma_{\text {beam }}$, the percentage of particles excited above this threshold is obtained. For a chromaticity of 2, 97.7\% of the recombined ions are excited above the threshold, while for a chromaticity of 4 this value is $75 \%$. This is assuming no effect due to space-charge tune spread.

A second limitting factor to using the AC to lose the recombined ions comes from IBS. Due to IBS the aperture will be completely filled. A small excitation of the fully stripped ion bunch will 
therefore causes losses at the tails of the distributions. As such, the signal obtained from the losses of $\mathrm{Au}^{78+}$ will be contaminated by losses of $\mathrm{Au}^{79+}$. No clear estimate was made to determine the losses of $\mathrm{Au}^{79+}$ due to the lack of knowledge on the form of the distribution at the wall edges due to IBS. Using the AC dipole to excite the recombined ions to large amplitudes and detect them at collimators does not provide an accurate and repeatable recombination rate monitor.

\subsection{Lattice with large dispersion $\&$ small $\beta$-function}

A lattice with large local dispersion is ideal to measure the produced $\mathrm{Au}^{78+}$ ions. The charge difference between the two populations of ions will cause the recombined ions to have a different closed orbit in large dispersive regions. By increasing the dispersion in a specific location the two ion populations can be separated. The $\mathrm{Au}^{78+}$ beam may then be captured and monitored, which would also directly function as integrator of the particle population.

Using the following beam parameters, the closed orbit separation in the nominal lattice in the arcs can be determined.

$$
\begin{aligned}
\epsilon & =0.6 \mu \mathrm{m} \\
\beta & =50 \mathrm{~m} \\
D & =1.8 \mathrm{~m} \\
\frac{\Delta q^{\prime}}{q^{\prime}} & =\frac{79-78}{78}=\frac{1}{78} \\
\sigma_{p} & =5 \cdot 10^{-4}
\end{aligned}
$$

The beam size is determined using:

$$
\sigma_{B}(s)=\sqrt{\epsilon \beta(s)+D(s) \sigma_{p}}=5.6 \mathrm{~mm}
$$

The transverse distance between the closed orbit of recombined ions and the nominal bunch is given by,

$$
\Delta C O=D \frac{\Delta q^{\prime}}{q^{\prime}}=23 \mathrm{~mm}=4.1 \sigma_{B}
$$

The nominal dispersion in the arcs is too small to separate the beams sufficiently. A local large dispersive section must be designed to further increase the beam separation. Figure 3.3 shows the resulting closed orbit deviation for the ranges of $\beta$ and dispersion functions that are of interest in the arcs. An ideal lattice contains a large dispersion and small $\beta$-function at a single location to create an as large as possible closed orbit separation.

The transitional jump $\left(\gamma_{T}\right)$ quadrupoles in the arcs may be used to create a local dispersion bump. The RHIC contains eight $\gamma_{T}$ quadrupoles per arc used to adjust the transition energy with dispersion deviations. Four of these quadrupoles are located near the arc centers in large dispersive sections, while the others are located in pairs at the ends of the arcs where the dispersion is smaller. Idealy these quadrupoles are separated by $90^{\circ}$, however, in RHIC the current phase advance between the quadrupoles is $83^{\circ}$. This puts considerable constraints on finding a lattice with large local dispersion without $\beta$ - and dispersion wave leakage. The $\gamma_{T}$ are of course limited in their powering strengths. The maximum strength of the $\gamma_{T}$ quadrupoles found in RampEditor are:

$$
\begin{aligned}
k & =6.3 \cdot 10^{-3} \mathrm{~m}^{-1} \quad(\text { at } \gamma=23) \\
& =3.52 \cdot 10^{-2} \mathrm{~m}^{-1} \quad(\text { scaled to } \gamma=4.1)
\end{aligned}
$$

Operational strength calculated from design manual (corrector quadrupoles) [18]: 


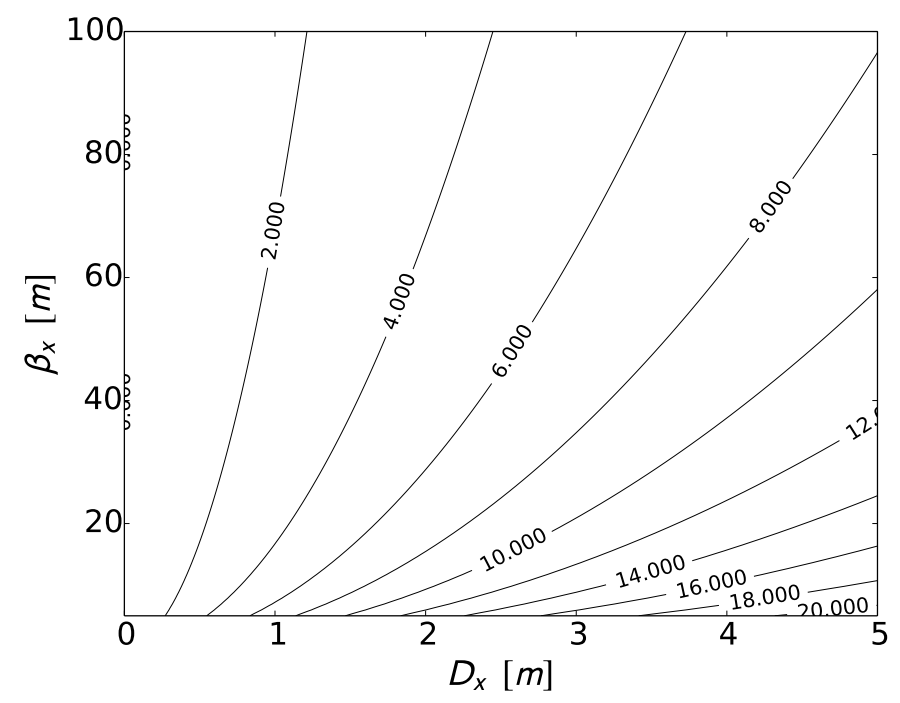

Figure 3.3: Closed orbit separation in $\sigma_{\text {beam }}$ for the range of interest of $\beta$ and dispersion functions.

$$
k=\int \frac{B^{\prime}}{B \rho} d l \equiv \frac{L_{e f f}}{B \rho} \frac{\Delta B_{y}}{\Delta x}=\frac{0.555}{35} \frac{0.067}{0.025}=4.2 \cdot 10^{-2} \mathrm{~m}^{-1}
$$

Note that the operational current of the $\gamma_{T}$ quadrupoles in the design manual is $49.8 \mathrm{~A}$, while its quench current is $190 \mathrm{~A}$. If needed the quadrupole strength may therefore be increased further.

The arc between IP12 and IP2 has been chosen to create a local dispersion bump with the $\gamma_{T}$ quadrupoles, to minimize the effects on the experiments and avoid the sensitive RF and DUMP regions. Though a fully closed optics distortion is preferable, arc 1 will allow for any leakage to be recovered in the IR. As both beams are independent and that the cryostats are separate in the arcs, it is not necessary to find a point with large dispersion and small $\beta$-function that works for both beams at the same location. Both cryostats will have to be opened, and the locations of these interventions are independent for both beams.

Due to the large number of constraints and the unfavorable phase advance between the cells all $8 \gamma_{T}$ quadrupoles in the arcs are used. The layout of the quadrupoles is presented in figure 3.4. Note that the layout of the quadrupoles is not symmetric in the arcs. The default powering scheme of the $\gamma_{T}$ quadrupoles is also shown in figure 3.4. The central four quadrupoles are powered together, while the outer four are also powered together. This is currently the default scheme, however, polarity switches or additional power supplies can be considered.

Attempts to find a lattice with large local dispersion and a small $\beta$ function in the arcs were unsuccesful using the current power scheme of the $\gamma_{T}$ quadrupoles. Various different powering schemes have been considered. Results indicate that a favorable lattice is achievable when powering the $\gamma_{T}$ quadrupoles in pairs. Figure 3.5 shows the proposed powering scheme. With this new powering setup and using the trim coils (TQ4, TQ5, TQ6) as well as, depending on the beam, the $8^{\text {th }}$ or $9^{\text {th }}$ focussing quadrupole of both insertion regions enclosing the arc it is possible to obtain a large enough dispersion wave in the arcs, while retaining a small $\beta$-function. The used quadrupoles in the IR and their powering scheme are shown in figure 3.6.

\subsubsection{Lattice for BLUE Beam}

A lattice is proposed for the blue beam that yields a maximal closed orbit separation of $7.4 \sigma_{B}$. This is largely sufficient to capture the recombined ions in the arcs. Figure 3.7 shows the achieved dispersion wave in the RHIC lattice, compared to the dispersion function in the original lattice. The 

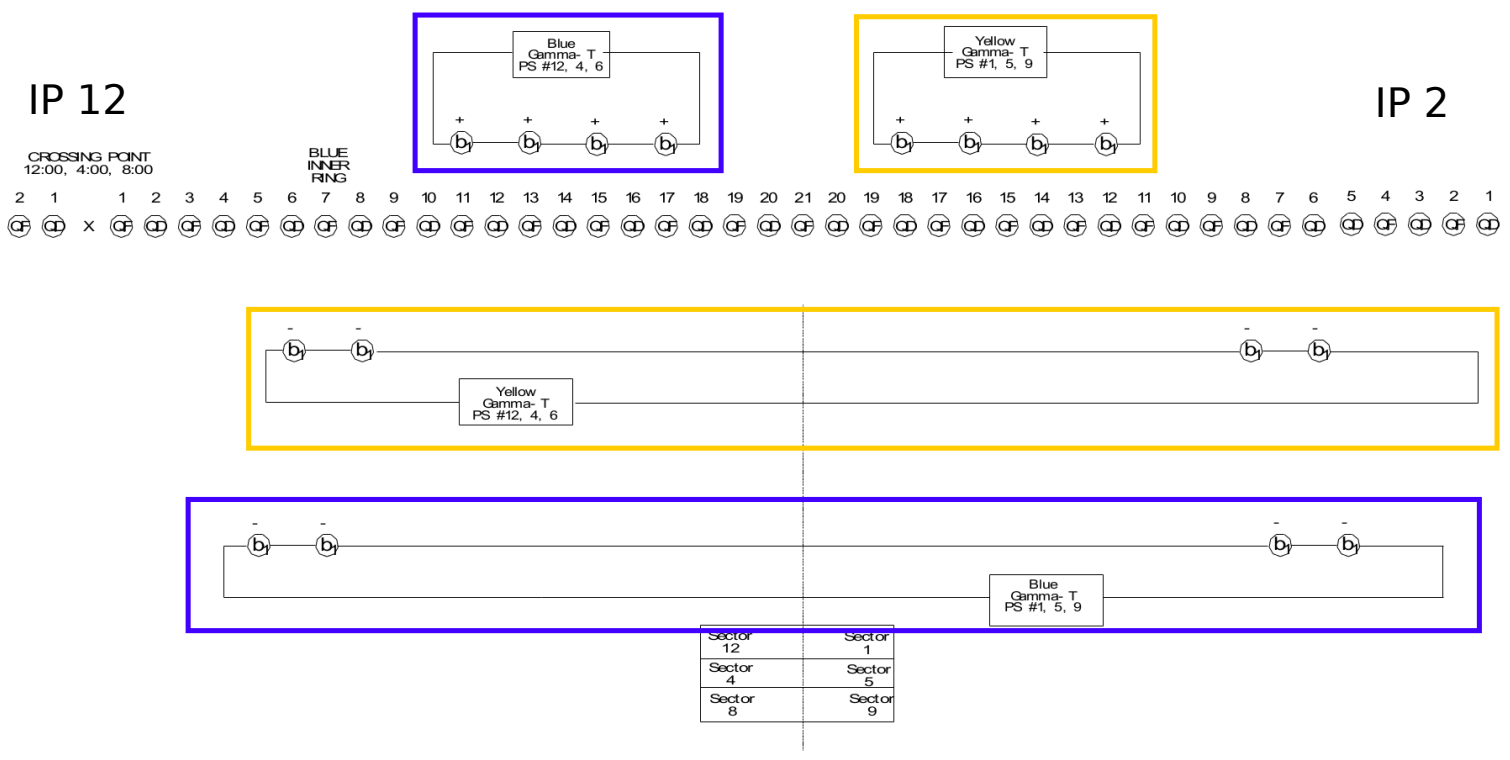

Figure 3.4: Current default powering scheme for the $\gamma_{T}$ quadrupoles in the arcs. There are two power supplies per beam per arc to power the quadrupoles. In the blue boxes the powering of the quadrupoles for the BLUE beam is shown. The powering for the YELLOW beam is shown in yellow.
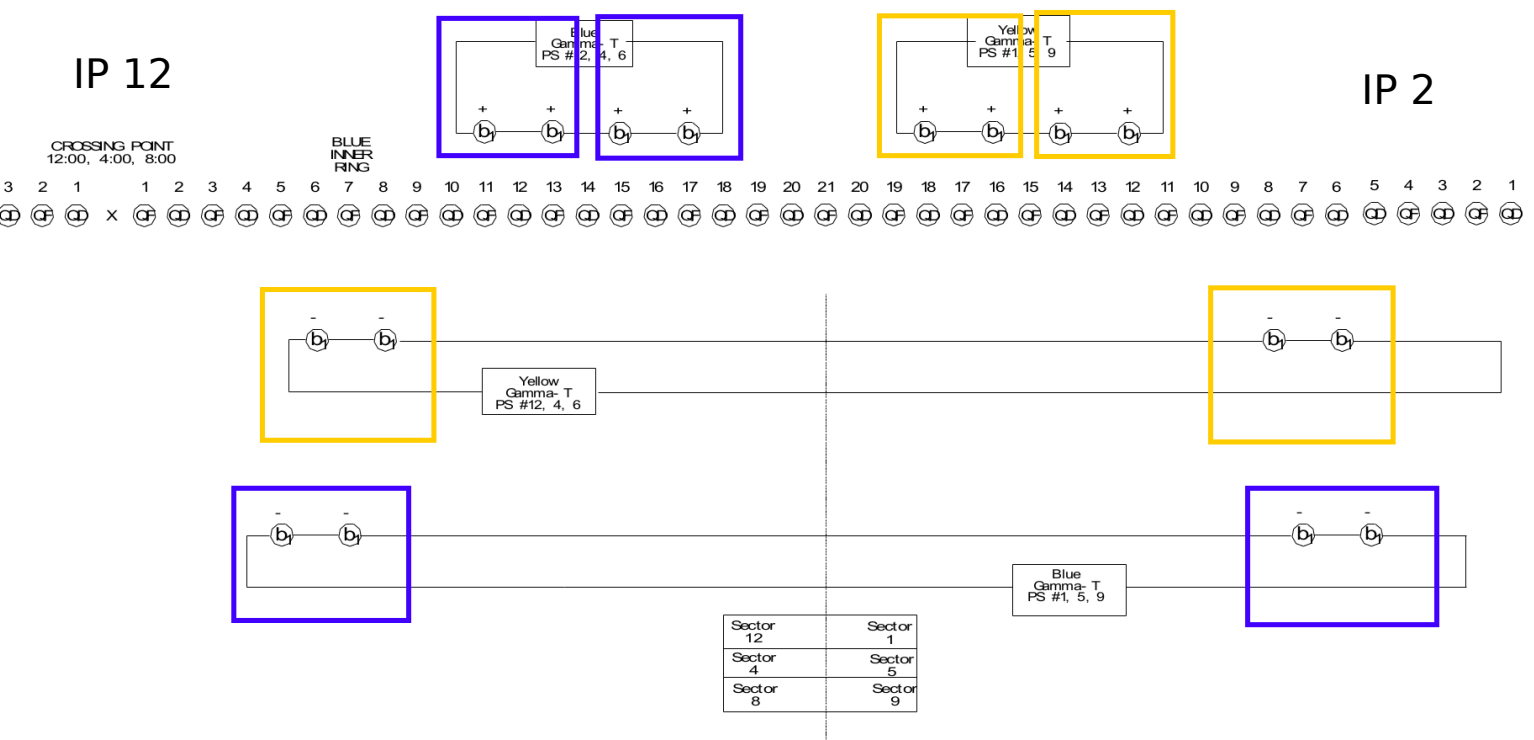

Figure 3.5: The proposed powering scheme to increase the flexibility for creating a dispersion wave in the arcs. The quadrupoles are now powered in pairs, which requires two additional power supplies per beam. The colors indicate the used beam. 


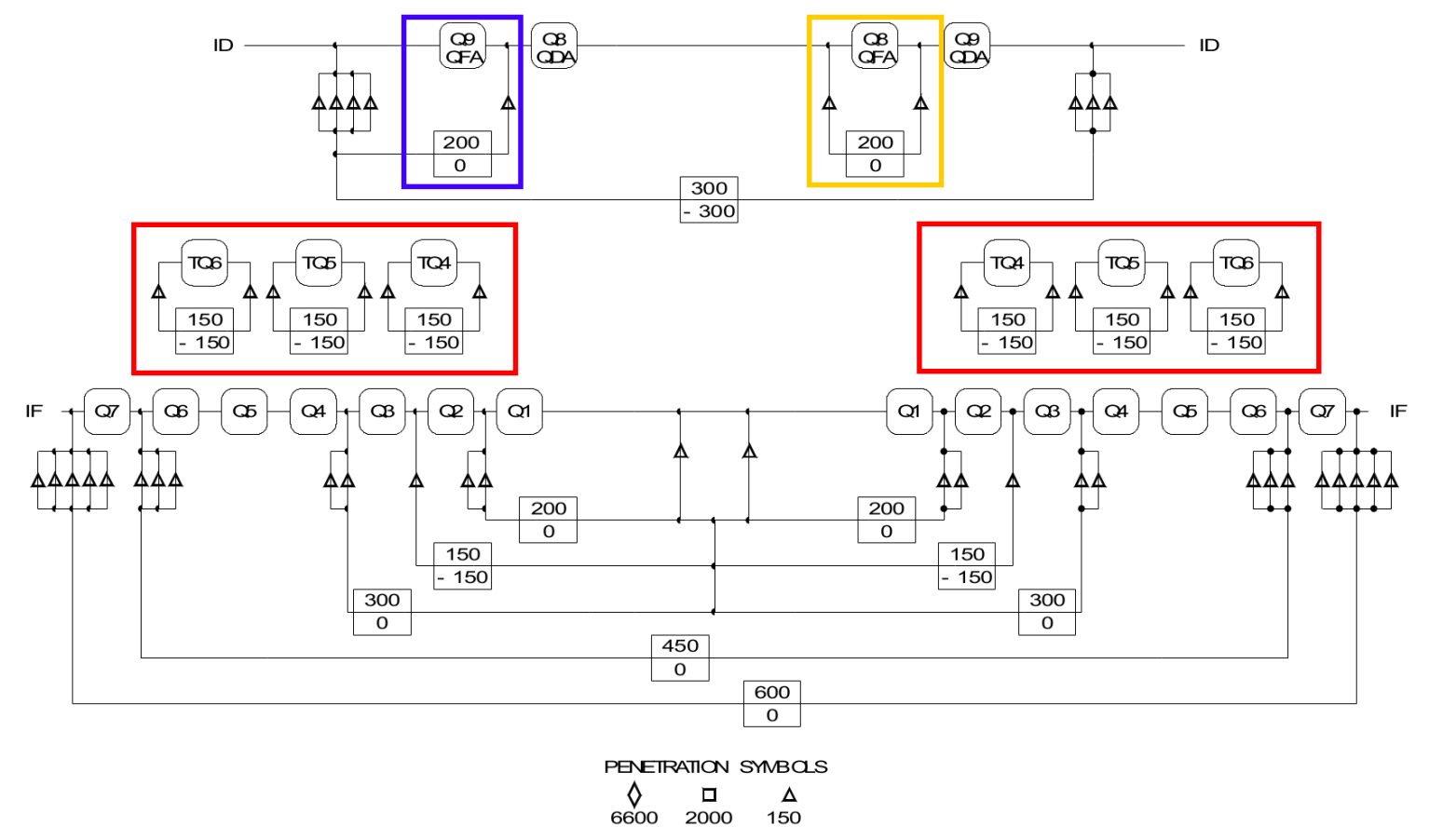

Figure 3.6: The powering scheme for the quadrupoles inside the insertion regions. The trim quadrupoles (TQ), shown in red, are used in both beams in both enclosing IR for fine tuning of the matching. The QF9 and QF8 are used for the BLUE and YELLOW beam respectively.

dispersion is increased by almost $1 \mathrm{~m}$ in BI12_QF13 and by $0.5 \mathrm{~m}$ in BI12_QD14. Furthermore, the produced wave closes at the end of the arc and there is no leakage throught the rest of the accelerator. The closed orbit separation in the arc is presented in figure 3.8. There is a clear maximum of 7.4 $\sigma_{B}$ near BI12_QD14.

The new lattice has an RMS $\beta_{X}$-beat and $\beta_{Y}$-beat of $32 \%$ and $13 \%$ respectively in the affected arc. The $\beta$-functions are presented in appendix B. All optical functions distortions close at the end of the arc and do not leak into the rest of the accelerator.

\subsubsection{Lattice for YELLOW Beam}

A comparable lattice has been obtained for the yellow beam, where the dispersion is increased by almost $1 \mathrm{~m}$ in YO1_QF14 and by $0.5 \mathrm{~m}$ in YO1_QD15, as shown in figure 3.9. These results yield a slightly smaller maximal closed orbit separation than obtained in the BLUE beam. The beam separation in the arcs is shown in figure 3.10. At $7 \sigma_{B}$ the closed orbit separation is still large enough for the detection of recombined ions. As well as for the blue beam, the dispersion bump closes nicely in the yellow beam.

The RMS $\beta$-beating in the horizontal and vertical planes are both at $13 \%$. Furthermore, the distortions close at the end of the arc, and there is almost no leakage to the rest of the accelerator.

\subsubsection{Detectors for Recombined Ions in the Arcs}

Detection of recombined ions in the arcs can be done with several methods that are either intrusive or non-intrusive. Non-intrusive detection consists of driving the beam of $\mathrm{Au}^{78+}$ into the physical wall. The ions will be lost in the wall thereby creating showers of secondary partiles that can be detected outside the cryostat using pin diodes. This method has been succesfully used before at high energies to detect recombined copper from bound free pair production [19]. Tests will be performed in the coming RHIC run to test the sensitivity at low energy Au operation. The sensitivity 

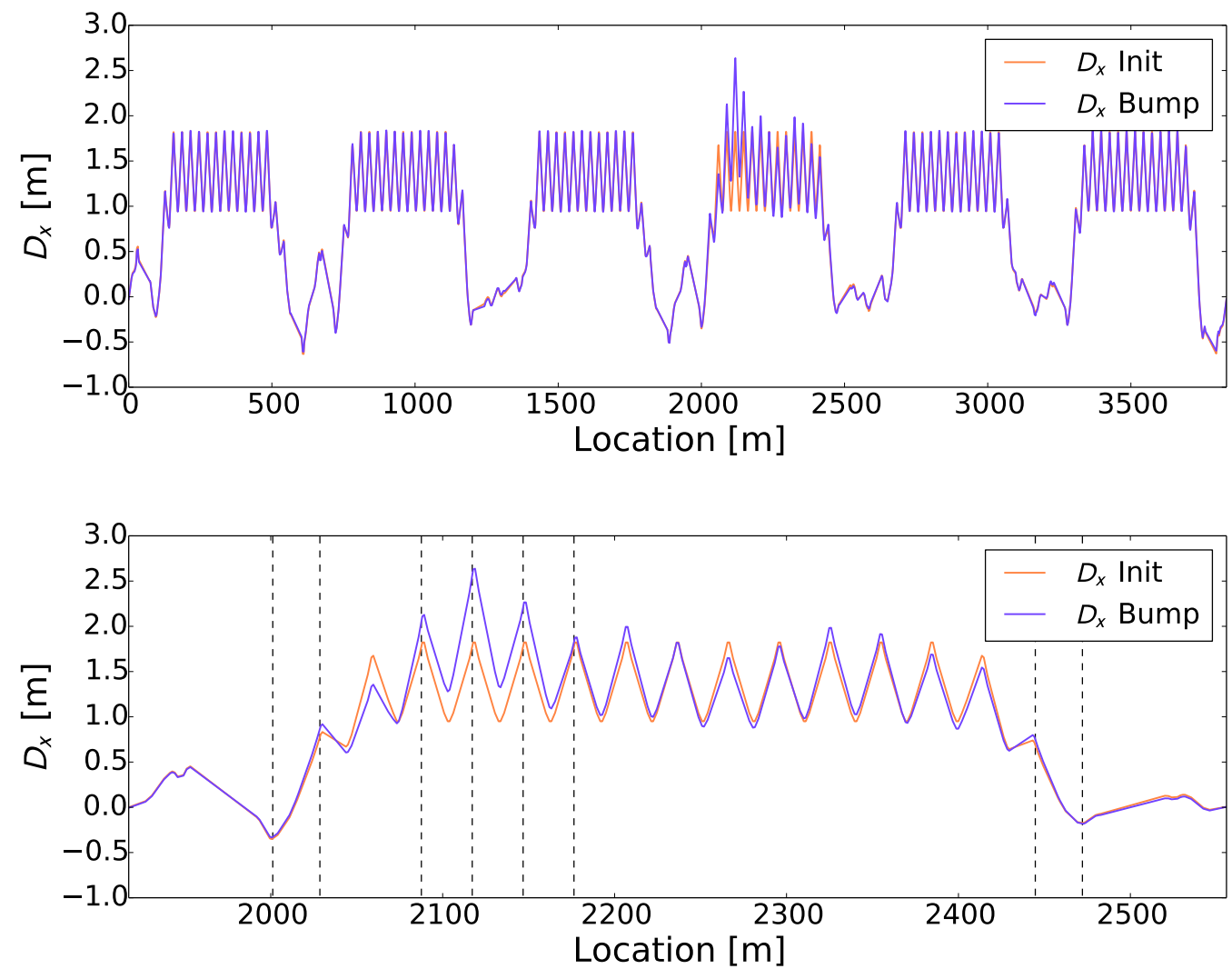

Figure 3.7: The horizontal dispersion for the initial lattice, in orange, and for the lattice with the dispersion wave, in blue, for the BLUE beam. The dispersion is increased by almost $1 \mathrm{~m}$ in BI12_QF13 and by 0.5m in BI12_QD14.. The created dispersion wave closes at the end of the arc, and no distortions are observed 

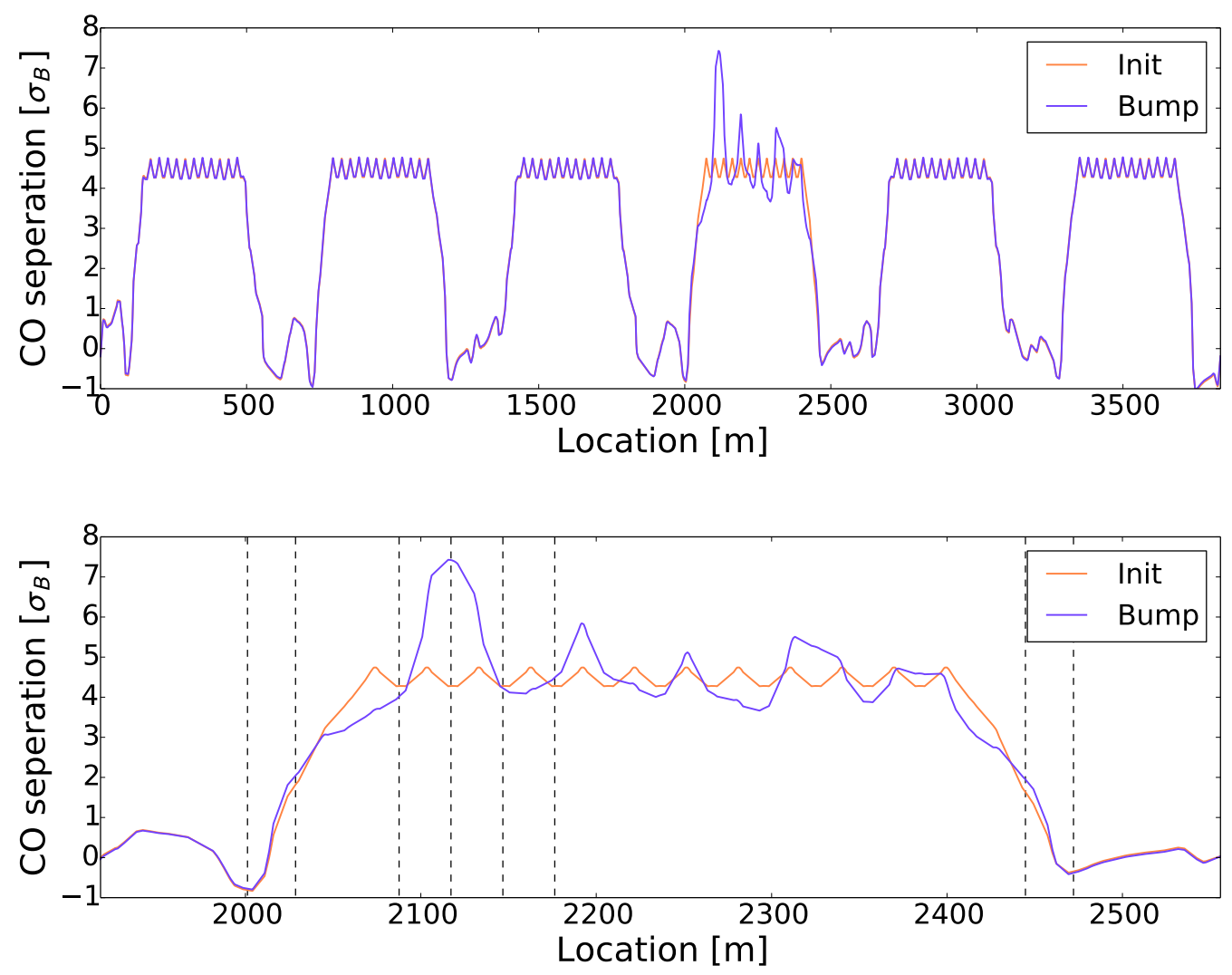

Figure 3.8: separation between the closed orbit of the fully stripped ions and the recombined ions. The upper figure shows the separation for the complete RHIC, while the lower figure shows the separation for the arc between IP12 and IP2. A maximum separation of $7.8 \sigma_{B}$ is obtained in the 

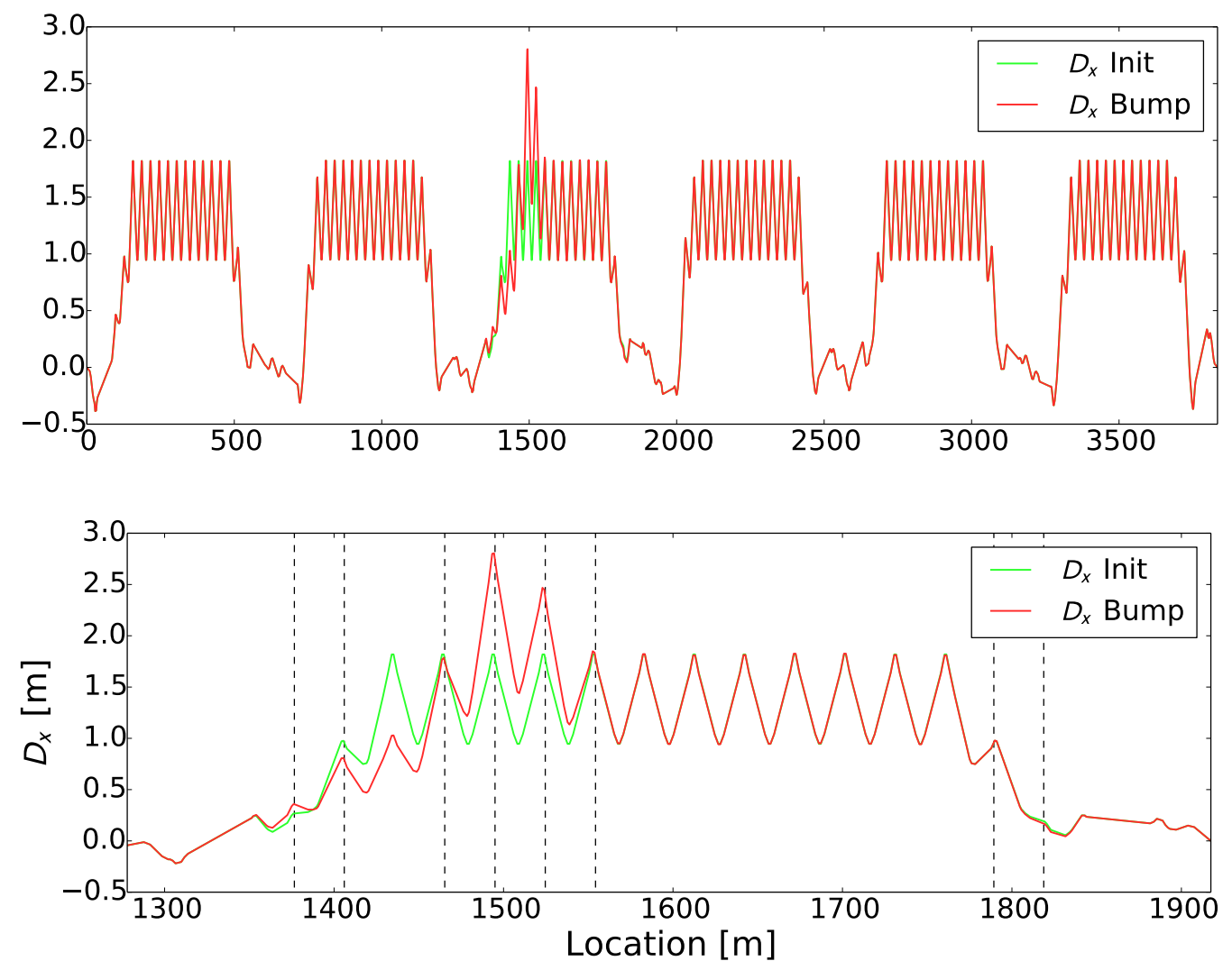

Figure 3.9: The horizontal dispersion for the initial lattice, in orange, and for the lattice with the dispersion wave, in blue, for the BLUE beam. The dispersion is increased by $1 \mathrm{~m}$ in QF15 and by $0.5 \mathrm{~m}$ near QD14. The created dispersion wave closes at the end of the arc, and no distortions are observed 

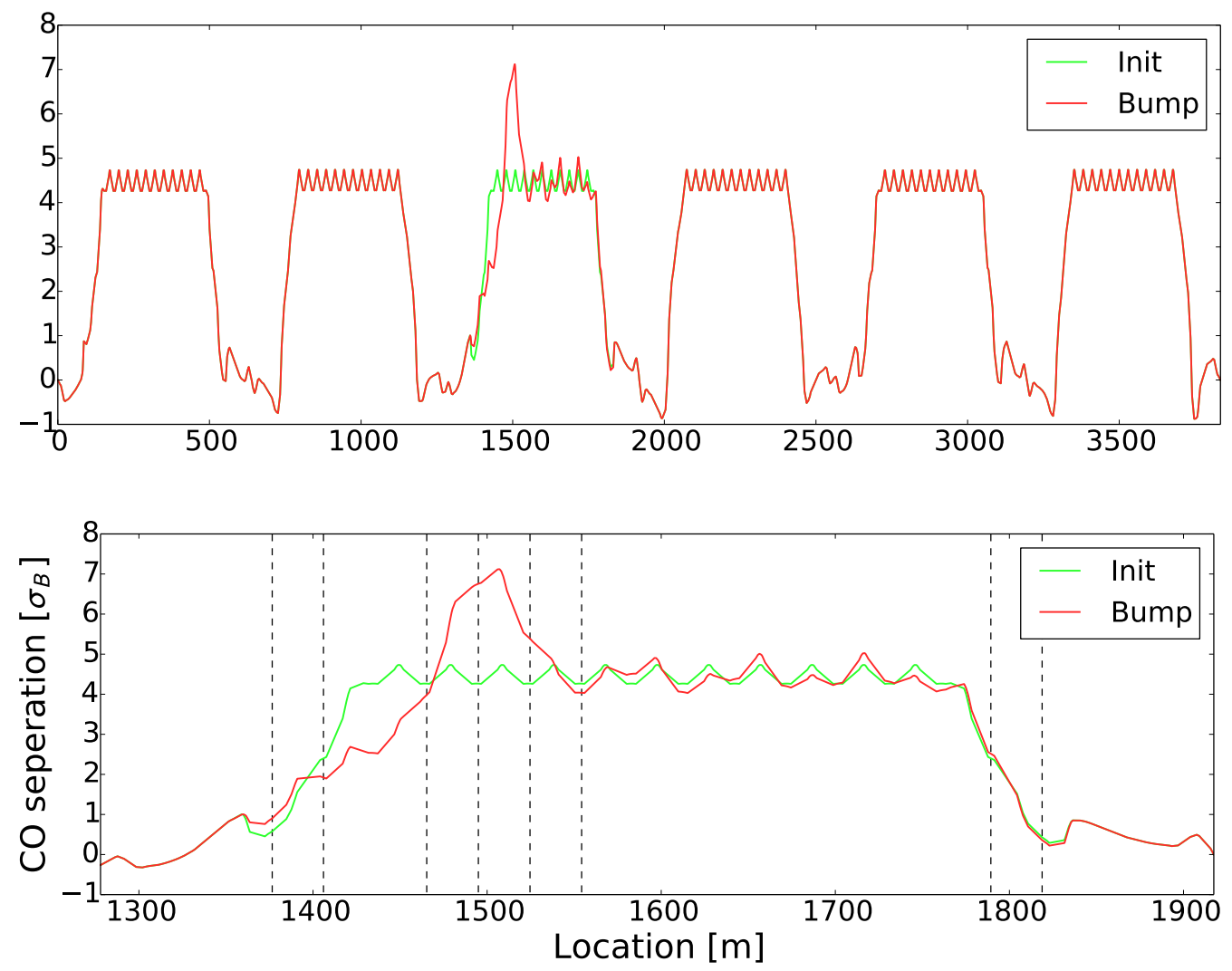

Figure 3.10: separation between the closed orbit of the fully stripped ions and the recombined ions. The upper figure shows the separation for the complete RHIC, while the lower figure shows the separation for the arc between IP12 and IP2. A maximum separation of $7.8 \sigma_{B}$ is obtained in the 
may be improved by increasing the number of pin diodes and correctly aiming them at the source of $\mathrm{Au}^{78+}$ losses.

Installing a collimator inside the cryostat to capture the recombined ions would localize the losses to a single point. The created showers can then be detected outside the cryostat using pin diodes. This, however, requires breaking open the cryostat to install the collimator. Another intrusive detection method consists of designing a roman pot detector that can insert a detector directly inside the beam pipe. This is the most direct way to detect the recombined ions, but also the most demanding method. A roman pot detector would have to be designed that fits inside the arcs, where space is limited. 


\section{Alternatives to Recombination Monitors}

This chapter presents alternative methods to recombination monitors of Au that are also considered. Though these were not the main focus of this study they have been included here to provide an exhaustive overview of methods available for electron cooling monitoring and tuning.

\subsection{Direct detection of Electron Cooling with Schottky System}

The main method for electron cooling detection as presented during the DOE review on 5-6 November 2015 is based on the Schottky spectra. Assuming an overlap between the electron and ion beams and a relative energy matching within $d E / E<0.1 \%$ it will be possible to detect the direct electron cooling effect in the Schottky spectra. A peak will start to develop at the electron energy. Energy tuning of the electron beam can then be done depending on the measured electron energy. Such a method based on a Schottky systems has been successfully used to detect cooling effects at Fermilab. Figure 4.1 shows the Schottky measurements performed at FermiLab to monitor electron cooling and tune the electron energy (S. Nagaitsev - FNAL).

At relative energy deviations of $d E / E<0.1 \%$ this procedure to monitor the electron cooling will be the preferred method. This method may be expanded to allow for electron cooling detection at larger energy deviations. By debunching the ion beams it is possible to fill the momentum aperture to approximately $1 \%$. This will enlarge the acceptance for the electron beam energy. However, the ion density will be considerably lower than before which will decrease the cooling. As suc, it will be more challenging to detect the electron energy.

\subsection{Recombination of Deuteron}

A second alternative procedure to measure the quality of the alignment between the electron beam and the ion beam is using alternative hadrons such as deuteron. Deuteron has a charge to mass ratio that is close to that of the gold ions. Recombination of deuteron yields a neutral deuterium particle that could be detected locally after the electron cooling. The recombination rate however will be much lower due to the $Z^{2}$ dependence of the cross-section. The recombination rates for deuteron may be calculated using the same method as for the $A u^{79+}$ recombination presented in chapter 2 . The results for the deuteron recombination rates as a function of relative energy deviation are presented in figure 4.2 .

The recombination rate coefficient $\alpha_{r}$ is approximately $10^{4}$ times smaller than for the gold ions, shown in figure 2.1. However, due to the charge differences the number of particles per bunch can be increased by almost 100. Furthermore, the detection of deuterium will be done locally. This could further increase the number of particles to be detected. Deuterium will interact with the magnets, cryogenics and shielding before exitting the accelerator ring. This will most likely produce 


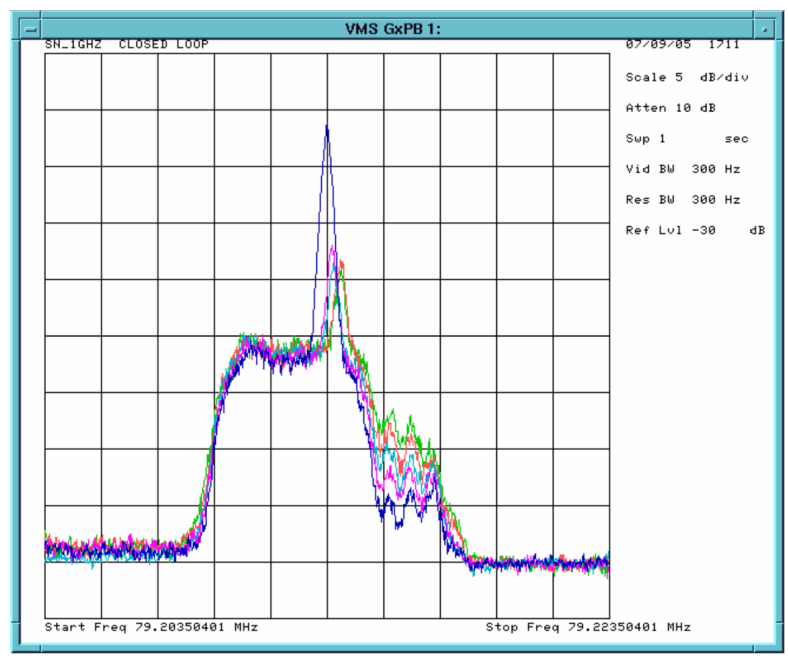

Figure 4.1: Schottky spectra showing the effect of electron cooling and the adjustment of the electron energies. (S. Nagaitsev - FNAL)
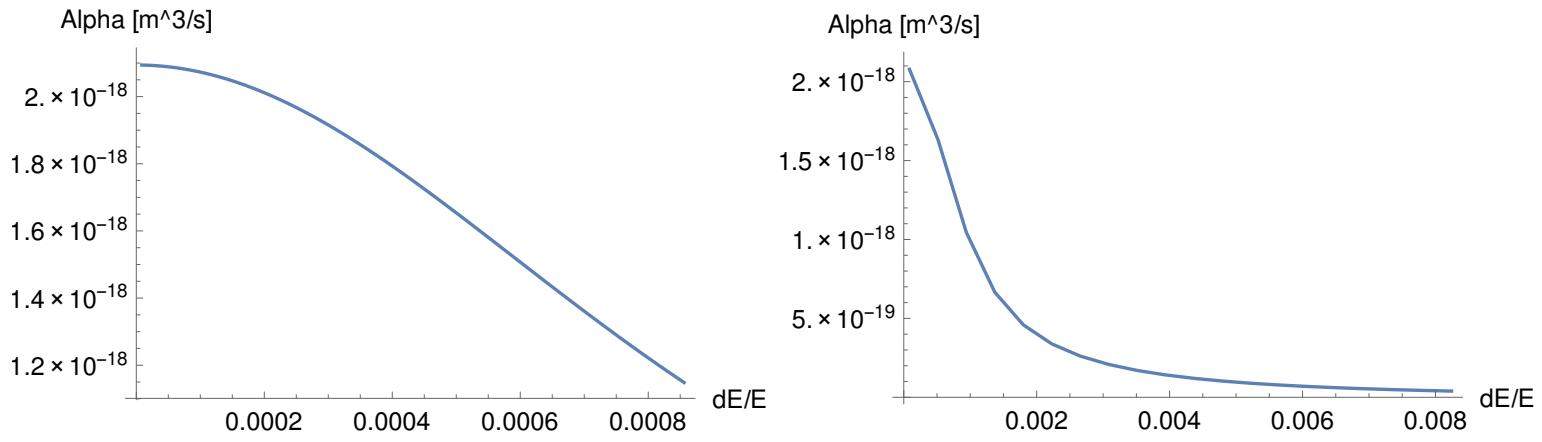

Figure 4.2: Recombination rate coefficients for deuteron $(Z=1)$ and electrons. The same beam properties are assumed for the deuteron bunch as for the gold ion bunches. 
showers of radiation. Such radiation may be measured using scintilators positioned outside the cryogenics. The advantage of such a detection method is that it would not be intrusive as well as cheap. However, there is still uncertainty as to what will come out of the dipoles. Studies on this topic and MCNPX simulations are currently performed by Kin Yip. 


\section{Conclusions}

Monitoring of recombination rates will provide a secondary tuning tool to approach optimal electron cooling conditions. The various available methods to monitor recombination rates in the electron cooler have been studied.

Detection of the change in revolution frequency between the fully stripped ions and the recombined ions using the Schottky system will not be possible. The change in revolution frequency is $2.4 \cdot 10^{-5}$ and is smaller than the frequency spread of the main ion beam $\sigma_{f}=2.9 \cdot 10^{-5}$. This signal will therefore not be discernable in the schottky spectrum.

Furthermore, the momentum of the ions does not change during the radiative recombination process. This means that the recombined ions will not leave the bucket into the abort gap. As such, the particles can not be extracted through resonant kicking in the abort gap.

The recombined ions will experience a tune shift due to the charge difference. For a first order chromaticity of 2 , the induced tune shift will be $\Delta Q=0.025$. The tune spread due to a momentum spread of $\sigma_{p}=5 \cdot 10^{-4}$ will be $\sigma_{p}=10^{-3}$. As such, the $\mathrm{Au}^{78+}$ ions can be excited independently using either the BTF or the AC-dipole. The tune composition can be determined using the BTF, and allows for low-intrusive monitoring. For the BTF, an excitation of $150 \mu \mathrm{m}$ is needed to obtain a clear spectrum for Au beams after 1 minute of operation. Such amplitudes are achievable with the current setup.

The AC dipole can be used to excite the ions to large amplitudes to lose the $\mathrm{Au}^{78+}$. The measured losses, however, will also contain a contribution of $\mathrm{Au}^{79+}$. Due to space-charge tune spread and unknown beam distributions due to IBS the amount of $\mathrm{Au}^{79+}$ lost through this method could not be clearly determined. Using the AC dipole to lose the $\mathrm{Au}^{78+}$ ions is therefore not the preferred method.

A lattice was found with increased dispersion in the arc between IP12 and IP2 to create a closed orbit separation between the two ion populations. A separation of 7.4 $\sigma_{B}$ and $7 \sigma_{B}$ was found for respectively the BLUE and YELLOW beam. These separations are sufficiently large to only detect $\mathrm{Au}^{78+}$ ions. The recombined ions can be lost in the beam pipe at a specific location. The produced showers can then be detected using aimed pin diodes located outside the cryostat. Tests will be performed in the upcoming RHIC run to determine the feasibility of this method. Detection may be improved by installing a collimator in the arc, or by designing a roman pot style detector to insert a detector in the beam pipe. These methods, however, require breaking open the cryostats.

Alternative methods, not based on the recombination of gold ions are currently considered. An alternative hadron, such as deuteron, may be used. The neutral deuterium created through recombination will leave the accelerator at the first dipole. This can function as a simple detector, however, rescalling of the calibrations will be needed to fit the gold beams.

Lastly, the cooling of the ion beam can be detecting in the Schottky spectra. This method is limitted to $d E / E<0.1 \%$ in normal operations. However, by debunching the ion beams the momen- 
tum acceptance can be increased to $1 \%$ thereby increasing the range available to detect cooling. This will require a dump and reinject operation scheme. 


\section{Appendix A}

The derivation for the recombination rates concisely presented in this appendix. The capture cross section is given by,

$$
\sigma=A\left(\frac{h v_{0}}{E}\right)\left[\ln \sqrt{\frac{h v_{0}}{E}}+0.1402+0.525\left(\frac{E}{h v_{0}}\right)^{\frac{1}{3}}\right]
$$

where $A=\frac{4}{3 \sqrt{3} \pi} \frac{h e^{2}}{\epsilon_{0} m_{e}^{2} c^{3}}=2.11 \cdot 10^{-22}$ and $h v_{0}=13.6 Z^{2} \mathrm{eV}$. The capture cross section may be expressed as a function of the transverse and longitudinal electron velocities by,

$$
\sigma=\frac{2 A h v_{0}}{m_{e}} \frac{1}{\left(v_{\|}^{2}+v_{\perp}^{2}\right)}\left[\frac{1}{2} \ln \frac{2 h v_{0}}{m_{e}}-\frac{1}{2} \ln \left(v_{\|}^{2}+v_{\perp}^{2}\right)+\gamma_{1}+\gamma_{2}\left(\frac{m_{e}}{2 h v_{0}}\right)^{\frac{1}{3}}\left(v_{\|}^{2}+v_{\perp}^{2}\right)^{\frac{1}{3}}\right]
$$

The recombination rate coefficient $\alpha_{r}$ is obtained by integration over the velocity distributions and the cross section.

$$
\alpha_{r}\left(v_{r e l}\right)=\int \sigma(v) v f_{e}\left(\mathbf{v}_{e}, v_{r e l}\right) f_{i}\left(\mathbf{v}_{i}, v_{r e l}\right) \mathrm{d}^{3} v
$$

Simplifying the constants as follows:

$$
\begin{aligned}
C_{0} & =\frac{2 A h v_{0}}{m_{e}} \\
C_{1} & =\frac{1}{2} \ln \frac{2 h v_{0}}{m_{e}}+\gamma_{1} \\
C_{2} & =\gamma_{2}\left(\frac{m_{e}}{2 h v_{0}}\right)^{\frac{1}{3}}
\end{aligned}
$$

The cross section then becomes:

$$
\begin{gathered}
\sigma=\frac{C_{0}}{\left(v_{\|}^{2}+v_{\perp}^{2}\right)}\left[C_{1}-\frac{1}{2} \ln \left(v_{\|}^{2}+v_{\perp}^{2}\right)+C_{2}\left(v_{\|}^{2}+v_{\perp}^{2}\right)^{\frac{1}{3}}\right] \\
f_{e}\left(\mathbf{v}_{e}, v_{r e l}\right)=\frac{m_{e}}{2 \pi k T_{e, \perp}} \exp \left(-\frac{m_{e} v_{e, \perp}^{2}}{2 k T_{e, \perp}}\right) \sqrt{\frac{m_{e}}{2 \pi k T_{e, \|}}} \exp \left(-\frac{m_{e}\left(v_{e, \|}-v_{r e l}\right)^{2}}{2 k T_{e, \|}}\right) \\
f_{i}\left(\mathbf{v}_{i}, v_{r e l}\right)=\frac{m_{i}}{2 \pi k T_{i, \perp}} \exp \left(-\frac{m_{i} v_{e, \perp}^{2}}{2 k T_{i, \perp}}\right) \sqrt{\frac{m_{i}}{2 \pi k T_{i, \|}}} \exp \left(-\frac{m_{i}\left(-v_{e, \|}+v_{r e l}\right)^{2}}{2 k T_{i, \|}}\right)
\end{gathered}
$$


where $v_{i}=-v_{e}$ was used. Temperatures are obtained from:

$$
\begin{aligned}
k T_{\|} & =m c^{2} \beta^{2}\left(\frac{\delta p_{\|}}{p}\right)^{2}=m\left(\Delta \nu_{\|}\right)^{2} \\
k T_{\perp} & =m c^{2} \beta^{2} \gamma^{2} \theta_{\perp}^{2}=\gamma^{2} m\left(\Delta v_{\perp}\right)^{2}
\end{aligned}
$$

The recombination rates are calculated from:

$$
\begin{aligned}
\alpha_{r}= & \frac{1}{\operatorname{Int}} \int_{-\infty}^{\infty} \int_{0}^{\infty}\left(v_{\|}^{2}+v_{\perp}^{2}\right)^{\frac{1}{2}} \sigma\left(v_{\perp}, v_{\|}\right) \cdot v_{\perp} \cdot \exp \left\{-\frac{m_{e}}{2 k}\left(\frac{v_{\perp}^{2}}{T_{e, \perp}}+\frac{\left(v_{\|}-v_{r e l}\right)^{2}}{T_{e, \|}}\right)\right\} . \\
& \exp \left\{-\frac{m_{i}}{2 k}\left(\frac{v_{\perp}^{2}}{T_{i, \perp}}+\frac{\left(v_{\|}-v_{r e l}\right)^{2}}{T_{i, \|}}\right)\right\} \cdot d v_{\perp} d v_{\|}
\end{aligned}
$$

where,

$$
\begin{aligned}
\text { Int }= & \int_{-\infty}^{\infty} \int_{0}^{\infty} v_{\perp} \cdot \exp \left\{-\frac{m_{e}}{2 k}\left(\frac{v_{\perp}^{2}}{T_{e, \perp}}+\frac{\left(v_{\|}-v_{r e l}\right)^{2}}{T_{e, \|}}\right)\right\} \\
& \exp \left\{-\frac{m_{i}}{2 k}\left(\frac{v_{\perp}^{2}}{T_{i, \perp}}+\frac{\left(v_{\|}-v_{r e l}\right)^{2}}{T_{i, \|}}\right)\right\} \cdot d v_{\perp} d v_{\|}
\end{aligned}
$$

and

$$
v_{r e l}=\frac{\left|v_{e, \|}-v_{i, \|}\right|}{1-\left(v_{e, \|} v_{i, \|}\right) / c^{2}}
$$

The expression given above for $\alpha_{r}$ has a singular point at $v_{\perp}=v_{\|}=0$. To avoid possible computational complications the expression is transformed to polar coordinates using:

$$
\begin{aligned}
v_{\perp} & =r \sin (\theta) \\
v_{\|} & =r \cos (\theta) \\
d v_{\perp} d v_{\|} & =r d r d \theta
\end{aligned}
$$

The recombination rate coefficient is then obtained with:

$$
\begin{aligned}
\alpha_{r}= & \frac{C_{0}}{I n t} \int_{0}^{\pi} \int_{0}^{\infty} r \cdot\left(C_{1}-\ln (r)+C_{2} r^{\frac{2}{3}}\right) \cdot \sin (\theta) \cdot \exp \left[-\frac{1}{2}\left(\frac{r^{2} \sin ^{2}(\theta)}{\left(\gamma^{2} \Delta v_{e, \perp}\right)^{2}}+\frac{\left(r \cos (\theta)-v_{r e l}\right)^{2}}{\left(\Delta v_{e, \|}\right)^{2}}\right)\right] \\
& \cdot \exp \left[-\frac{1}{2}\left(\frac{r^{2} \sin ^{2}(\theta)}{\left(\gamma^{2} \Delta v_{i, \perp}\right)^{2}}+\frac{\left(r \cos (\theta)-v_{r e l}\right)^{2}}{\left(\Delta v_{i, \|}\right)^{2}}\right)\right] d r d \theta \\
\text { Int }= & \int_{0}^{\pi} \int_{0}^{\infty} r^{2} \cdot \sin (\theta) \cdot \exp \left[-\frac{1}{2}\left(\frac{r^{2} \sin ^{2}(\theta)}{\left(\gamma^{2} \Delta v_{\perp}\right)^{2}}+\frac{\left(r \cos (\theta)-v_{r e l}\right)^{2}}{\left(\Delta v_{\|}\right)^{2}}\right)\right] \\
& \cdot \exp \left[-\frac{1}{2}\left(\frac{r^{2} \sin ^{2}(\theta)}{\left(\gamma^{2} \Delta v_{i, \perp}\right)^{2}}+\frac{\left(r \cos (\theta)-v_{r e l}\right)^{2}}{\left(\Delta v_{i, \|}\right)^{2}}\right)\right] d r d \theta
\end{aligned}
$$




\section{$\mathrm{B}$}

\section{Appendix B}

This appendix presents the obtained values from the matching of the dispersion wave and the $\beta$ functions for both beams. 


\begin{tabular}{|c|c|c|c|c|}
\hline BLUE & & & & \\
\hline Variable & Value & Initial Strength & Lower lim. & Upper lim. \\
\hline psiqt4_ir12 & $5.55378 e+00$ & $5.16861 e+00$ & $-1.0 e+02$ & $1.0 e+02$ \\
\hline psiqt5_ir12 & $2.15194 e+00$ & $2.62428 e+00$ & $-1.0 e+02$ & $1.0 e+02$ \\
\hline psiqt6_ir 12 & $1.93146 e-01$ & $4.75905 e-01$ & $-1.0 e+02$ & $1.0 e+02$ \\
\hline psqfa_ir 12 & $2.47471 e+01$ & $9.70024 e+00$ & $5.0 e+00$ & $1.5 e+02$ \\
\hline$k 1 l \_q g t$ out & $8.71801 e-03$ & $0.00000 e+00$ & $-1.0 e+20$ & $1.0 e+20$ \\
\hline$k 1 l \_q g t$ out 2 & $5.36768 e-03$ & $0.00000 e+00$ & $-1.0 e+20$ & $1.0 e+20$ \\
\hline$k 1 l \_q g t \_b u m p$ & $-4.12421 e-03$ & $0.00000 e+00$ & $-1.0 e+20$ & $1.0 e+20$ \\
\hline$k 1 l \_q g t \_b u m p 2$ & $-7.07346 e-03$ & $0.00000 e+00$ & $-1.0 e+20$ & $1.0 e+20$ \\
\hline psqfa_ir2 & $5.00000 e+00$ & $5.49920 e+00$ & $5.0 e+00$ & $1.5 e+02$ \\
\hline psiqt6_ir2 & $-1.09626 e+00$ & $-1.05329 e+00$ & $-1.0 e+02$ & $1.0 e+02$ \\
\hline psiqt5_ir2 & $-2.49590 e+00$ & $-2.35199 e+00$ & $-1.0 e+02$ & $1.0 e+02$ \\
\hline psiqt4_ir2 & $1.13825 e+01$ & $1.06475 e+01$ & $-1.0 e+02$ & $1.0 e+02$ \\
\hline \multicolumn{5}{|l|}{ YELLOW } \\
\hline Variable & Value & Initial Strength & Lower lim. & Upper lim. \\
\hline psoqt4_ir12 & $-1.24461 e+01$ & $-1.30386 e+01$ & $-1.0 e+02$ & $1.0 e+02$ \\
\hline psoqt5_ir12 & $-9.42158 e-01$ & $-3.26124 e-01$ & $-1.0 e+02$ & $1.0 e+02$ \\
\hline psoqt6_ir12 & $-2.05088 e+00$ & $-2.06409 e+00$ & $-1.0 e+02$ & $1.0 e+02$ \\
\hline psqfb_ir12 & $5.83649 e+00$ & $5.97293 e+00$ & $5.0 e+00$ & $1.5 e+02$ \\
\hline$k 1 l \_q g t \_o u t 1$ & $7.29401 e-04$ & $0.00000 e+00$ & $-4.0 e-02$ & $4.0 e-02$ \\
\hline$k 1 l \_q g t$ out 2 & $1.61385 e-02$ & $0.00000 e+00$ & $-4.0 e-02$ & $4.0 e-02$ \\
\hline$k 1 l \_q g t \_b u m p 1$ & $-7.43969 e-03$ & $0.00000 e+00$ & $-4.0 e-02$ & $4.0 e-02$ \\
\hline$k 1 l \_q g t \_b u m p 2$ & $-2.51589 e-03$ & $0.00000 e+00$ & $-4.0 e-02$ & $4.0 e-02$ \\
\hline psqfb_ir2 & $8.76854 e+00$ & $5.6520 e+00$ & $5.0 e+00$ & $1.5 e+02$ \\
\hline psoqt6_ir2 & $-2.30883 e+00$ & $1.26392 e+00$ & $-1.0 e+02$ & $1.0 e+02$ \\
\hline psoqt5_ir2 & $2.77486 e+00$ & $-9.20574 e+00$ & $-1.0 e+02$ & $1.0 e+02$ \\
\hline psoqt4_ir2 & $-1.15371 e+01$ & $9.00896 e+00$ & $-1.0 e+02$ & $1.0 e+02$ \\
\hline
\end{tabular}

Table B.1: Obtained values for dispersion wave. 

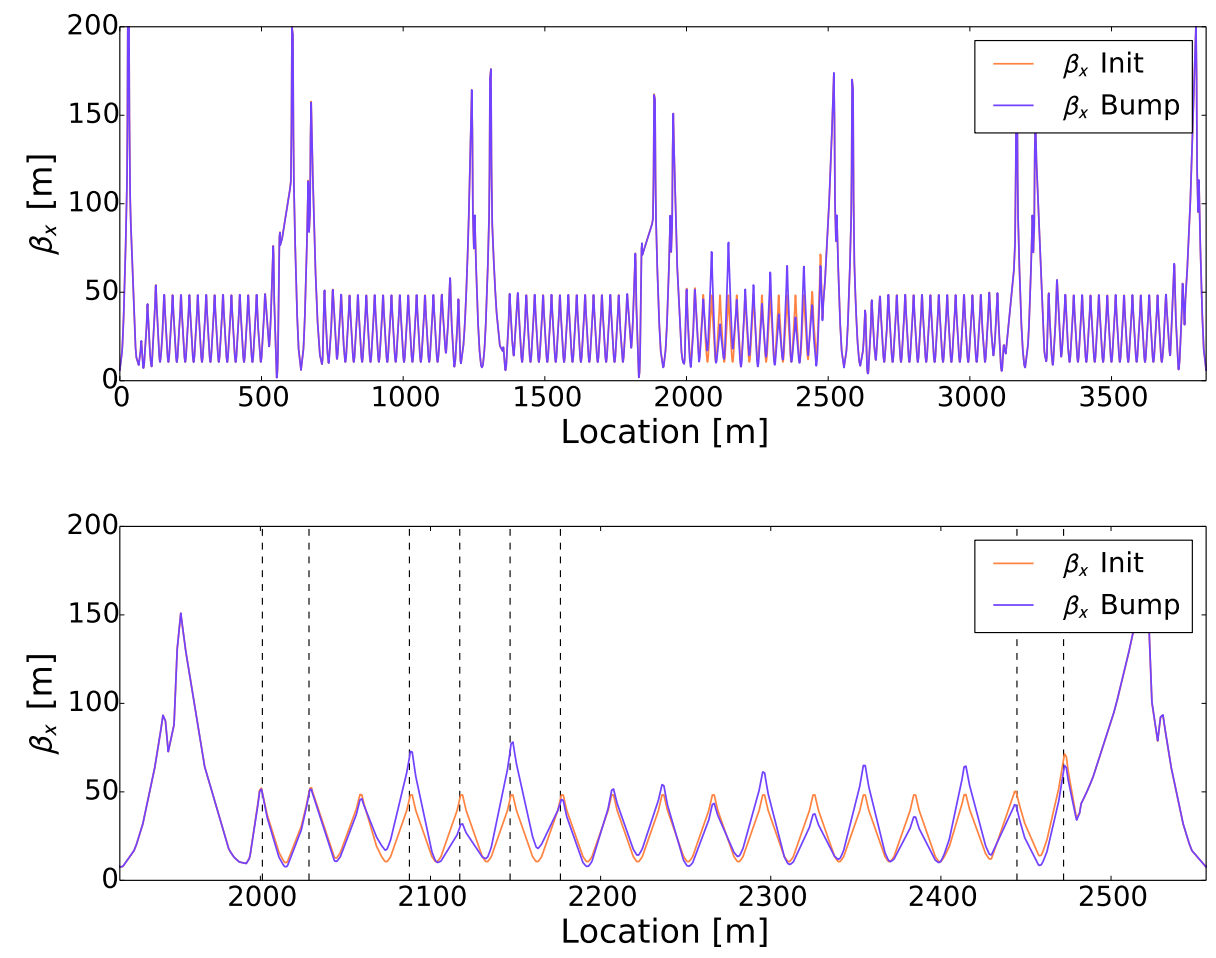

Figure B.1: Horizontal $\beta$-functions for the BLUE beam.
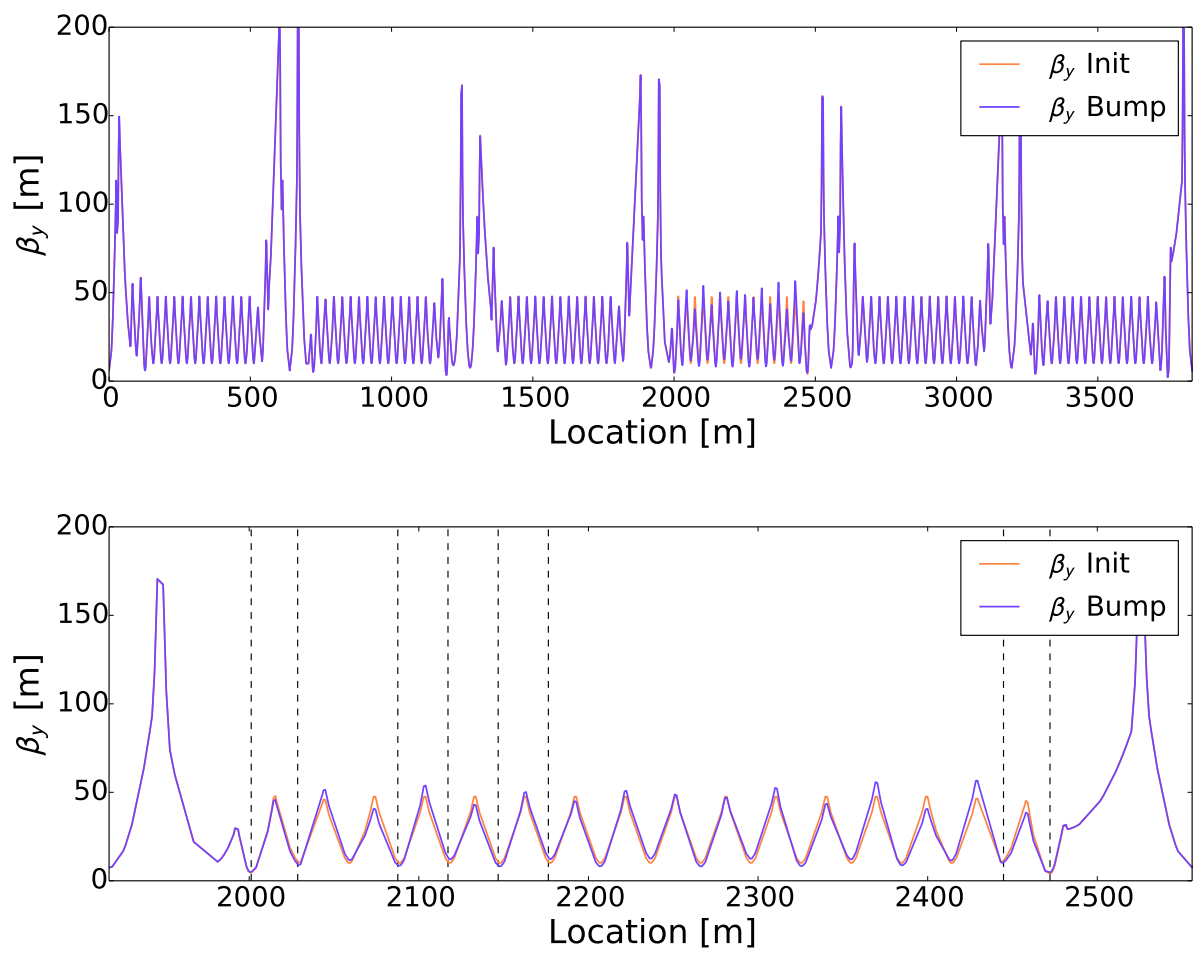

Figure B.2: Vertical $\beta$-functions for the BLUE beam. 

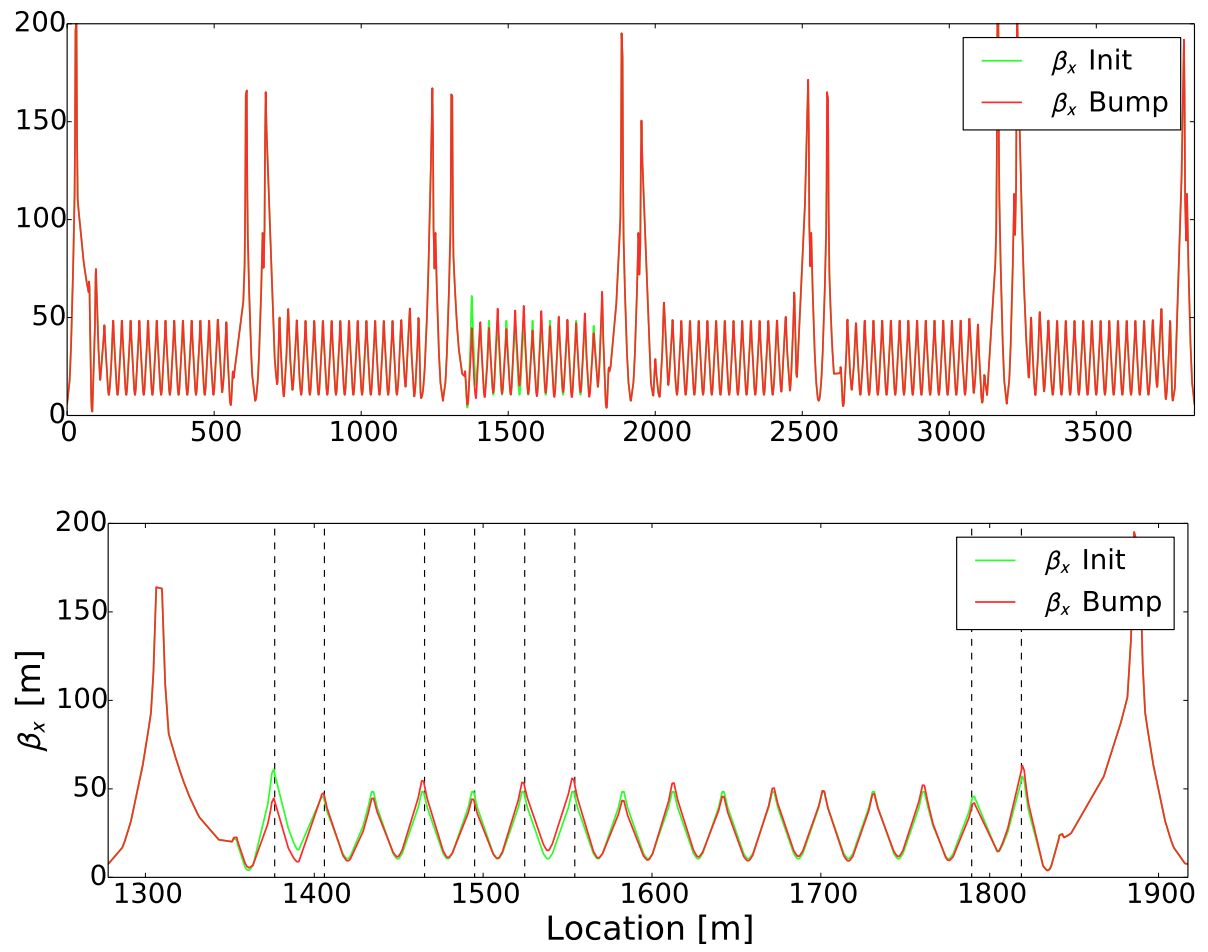

Figure B.3: Horizontal $\beta$-functions for the YELLOW beam.
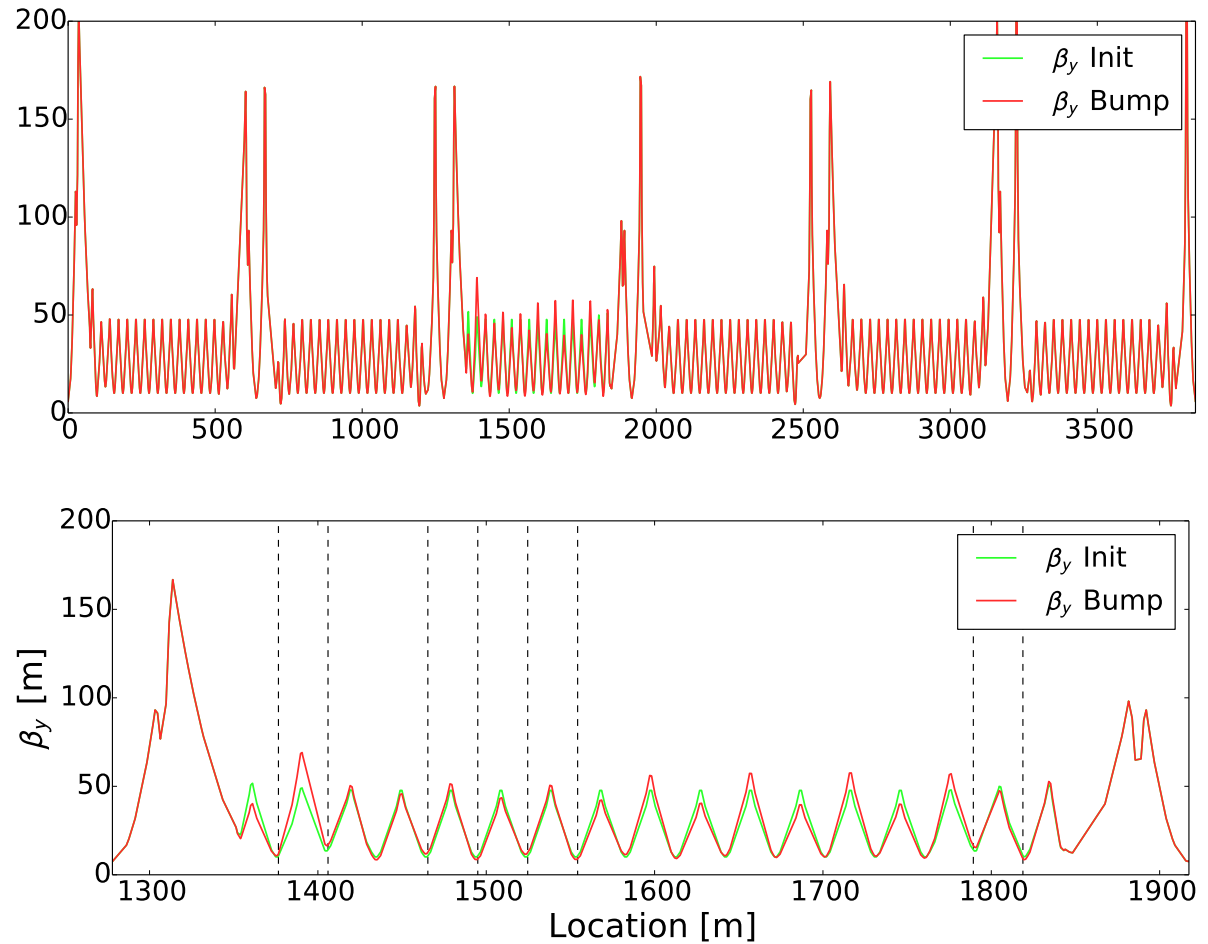

Figure B.4: Vertical $\beta$-functions for the YELLOW beam. 


\section{Bibliography}

[1] M. Stephanov, K. Rajagopal, and E. Shuryak, Signatures of the tricritical point in qcd, Phys. Rev. Lett. 81, 4816 (1998).

[2] Can we discover qcd critical point at RHIC? (BNL, 2006) RIKEN BNL Research Center Report No. BNL-75692-2006.

[3] A. Cho, Scheme for Boiling Nuclear Matter Gathers Steam at Accelerator Lab, Science 312, 190 (2006).

[4] G. S. F. Stephans, critrhic: the rhic low energy program, Journal of Physics G: Nuclear and Particle Physics 32, S447 (2006).

[5] M. Bell and J. S. Bell, Capture of cooling electrons by cool protons, Part. Accel. 12, 49 (1981).

[6] A. Wolf, G. Gwinner, J. Linkemann, A. A. Saghiri, M. Schmitt, D. Schwalm, M. Grieser, M. Beutelspacher, T. Bartsch, C. Brandau, A. Hoffknecht, A. Müller, S. Schippers, O. Uwira, and D. W. Savin, Recombination in electron coolers, Nuclear Instruments and Methods in Physics Research A 441, 183 (2000).

[7] A. Kuznetsov, I. Meshkov, and A. Philippov, Radiative recombination of ions and nuclei in electron cooling systems, Physics of Particles and Nuclei Letters 9, 346 (2012).

[8] W. Shi, S. Böhm, C. Böhme, C. Brandau, A. Hoffknecht, S. Kieslich, S. Schippers, A. Müller, C. Kozhuharov, F. Bosch, B. Franzke, P. Mokler, M. Steck, T. Stöhlker, and Z. Stachura, Recombination of $u 92+$ ions with electrons, The European Physical Journal D - Atomic, Molecular, Optical and Plasma Physics 15, 145 (2001).

[9] C. Montag, The LEReC Recombination Monitor, LEReC meeting.

[10] A. Fedotov, LEReC overview and status, (2015), RHIC Meeting.

[11] C. Scheidenberger, T. Stöhlker, W. Meyerhof, H. Geissel, P. Mokler, and B. Blank, Charge states of relativistic heavy ions in matter, Nuclear Instruments and Methods in Physics Research Section B: Beam Interactions with Materials and Atoms 142, 441 (1998).

[12] Homepage LISE++, http://lise.nscl.msu.edu/lise.html, .

[13] B. Franzke, IEEE NS-28 (1981) 2116, .

[14] S. Y. Lee, Accelerator physics; 3rd ed. (World Scientific, Singapore, 2012).

[15] M. Minty, A. curcio, C. Dawson, C. Degen, Y. Luo, G. Marr, B. Martin, A. Marusic, and K. Mernick, High precision tune and coupling feedback and beam transfer function measurements in rhic, in Proceedings of IPAC 2010, Kyoto, Japan (2010) pp. 522-524.

[16] A. Chao, Physics of Collective Beam Instabilities in High Energy Accelerators, Wiley Series in Beam Physics and Accelerator Technology (Wiley, 1993). 
[17] M. Bai, S. Y. Lee, J. W. Glenn, H. Huang, L. Ratner, T. Roser, M. J. Syphers, and W. van Asselt, Experimental test of coherent betatron resonance excitations, Phys. Rev. E 56, 6002 (1997).

[18] RHIC Configuration Manual (2006).

[19] R. Bruce, J. M. Jowett, S. Gilardoni, A. Drees, W. Fischer, S. Tepikian, and S. R. Klein, Observations of beam losses due to bound-free pair production in a heavy-ion collider, Phys. Rev. Lett. 99, 144801 (2007). 